\title{
KIC 7385478: An Eclipsing Binary with a $\gamma$ Doradus Component
}

\author{
Orkun Özdarcan and Hasan Ali Dal \\ Science Faculty, Department of Astronomy and Space Sciences, Ege University, 35100 Bornova, Izmir, Turkey \\ Email: orkun.ozdarcan@ege.edu.tr
}

(RECEIVED February 7, 2017; ACCEPTED March 20, 2017)

\begin{abstract}
We present spectroscopic and photometric analysis of the eclipsing binary KIC 7385478. We find that the system is formed by F1V + K4III-IV components. Combining results from analysis of spectroscopic data and Kepler photometry, we calculate masses and radii of the primary and the secondary components as $M_{1}=1.71 \pm 0.08 \mathrm{M}_{\odot}, M_{2}=0.37 \pm 0.04$ $\mathrm{M}_{\odot}$ and $R_{1}=1.59 \pm 0.03 \mathrm{R}_{\odot}, R_{2}=1.90 \pm 0.03 \mathrm{R}_{\odot}$, respectively. Position of the primary component in $\mathrm{HR}$ diagram is in the region of $\gamma$ Doradus type pulsators and residuals from light curve modelling exhibit additional light variation with a dominant period of $\sim 0.5 \mathrm{~d}$. These are clear evidences of the $\gamma$ Doradus type pulsations on the primary component. We also observe occasional increase in amplitude of the residuals, where the orbital period becomes the most dominant period. These may be attributed to the cool star activity originating from the secondary component.
\end{abstract}

Keywords: (stars:) binaries: eclipsing - stars: fundamental parameters - stars: individual (KIC 7385478) - stars: oscillations (including pulsations)

\section{INTRODUCTION}

High precision optical photometry from space telescopes is a milestone for modelling of eclipsing binary systems, where current models can not reproduce very small amplitude variations adequately. Even though, reasonable light curve modelling of these systems provides not only absolute physical properties, but also reveals additional small amplitude light variations beside the eclipse events and binarity effects (i.e. reflection, gravity darkening, etc.). This leads us to find accurate position of the components of these system on HR diagram and evaluate their variation nature more accurately.

There is a few indicators to reveal the internal layers of the stars. One of them is the stellar pulsation. Unfortunately, the pulsations can not be observable for each star. On the other hand, the initial analysis and some studies in the literature, such as Uytterhoeven et al. (2011), indicated that KIC 7385478 is one of the candidates for the eclipsing binaries with pulsating component. Moreover, the pulsation behaviour seen in the stars is very important pattern to understand the stellar itself and its evolution. According to the observations lasting as long as several decades indicate that there are several type pulsating stars such as Cepheid, $\gamma$ Doradus, and $\delta$ Scuti type pulsating stars in the Instability Strip in the Hertzsprung-Russell diagram, especially on the main sequence. All these types are separated by their locations in the Instability Strip from each other. Analysing the pulsation frequencies, which is generally known as stellar seismology called asteroseismology, the physical processes behind both the pulsating nature and stellar interiors can be revealed. This is why the pulsating stars have an important role to understanding stellar evolution (Cunha et al. 2007; Aerts Christensen-Dalsgaard).

KIC 7385478 is an interesting eclipsing binary whose out-of-eclipse variation does not seem regular. The system was identified as a variable star in ASAS catalogue (ASAS J195058+4259.8) for the first time (Pigulski et al. 2009) with a variation period of $1^{d} .6551$ and $V-I$ colour of $0^{m} .661$. Variation amplitude in $V$ and $I$ filters are given as $0^{m} .12$ and $0^{m} .19$, respectively. Later, Slawson et al. (2011) compiled the Kepler eclipsing binary catalogue ${ }^{1}$, including KIC 7385478. Extracted high precision light curves, ephemeris, period, and effective temperature estimation for most of the system are provided in the catalogue. The catalogue provides the effective temperature of KIC 7385478 as 6477 K. Pinsonneault et al. (2012) revised the temperature to $6735 \mathrm{~K}$. More recently, Armstrong et al. (2014) estimated the effective temperatures of the primary and the secondary components of KIC 7385478 as 6346 and $4719 \mathrm{~K}$, respectively, which are based on spectral energy distribution fitting. The ephemeris and the period were revised by Borkovits et al. (2016), who provided eclipse timing analysis of the system. Their analysis revealed the orbit of a third body physically bound to the eclipsing binary and they estimated the minimum mass

\footnotetext{
${ }^{1}$ http://keplerebs.villanova.edu/
} 
of the third body as $0.27 \mathrm{M}_{\odot}$. Beyond these studies, there is no comprehensive analysis of the system published so far.

In this study, we focus on spectroscopic and photometric modelling of the system. In the next section, we give summary of photometric observations, and spectroscopic observations including reduction process. Section 3 comprises spectroscopic, orbital, and light curve modelling together with calculated physical properties and evolutionary status of the system. In addition, we further give analysis on residuals from light curve modelling in order to investigate out-of-eclipse light variations. In the final section, we summarise and discuss our findings.

\section{OBSERVATIONS AND DATA REDUCTIONS}

\subsection{Kepler photometry}

There is no 'true' photometric filters in photometer of the Kepler space telescope, hence Kepler photometry contains no colour information. However, response function of the photometer covers a very broad wavelength range, which is between 4100 and $9100 \AA$ and this allows to collect photons from a large part of the optical spectrum and increases the photometric precision, thus enables to detect light variations with a sub-milimag amplitude, such as planet transit and small amplitude oscillations. Photometric measurements are collected in short cadence and long cadence mode, which have typical exposure times of $58.89 \mathrm{~s}$ and $29.4 \mathrm{~min}$, respectively. In this study, we use long cadence (29.4 min) data of KIC 7385478 available at Kepler eclipsing binary catalogue. We consider detrended and normalised fluxes (Prša et al. 2011; Slawson et al. 2011) in the catalogue. However, observations from quarter 12 and 13 are not included in the catalogue data file; hence, we downloaded data files of these missing quarters from $\mathrm{MAST}^{2}$ data archive centre, in fits format. In order to evaluate these data together with the catalogue data, we consider simple aperture photometry (SAP) measurements in the fits data files of quarter 12 and 13. Then we detrend and normalise SAP fluxes as described in Slawson et al. (2011). The final data set covers $\sim 4$ yrs of time span with 65722 data points in total. MAST archive reports $0.9 \%$ contamination level in the measurements, which indicates negligible contribution to the measured fluxes of KIC 7385478, if any.

\subsection{Spectroscopy}

We carried out spectroscopic observations of KIC 7385478 by $1.5-\mathrm{m}$ Russian-Turkish telescope equipped with Turkish Faint Object Spectrograph Camera (TFOSC) at Tubitak National Observatory ${ }^{3}$. Spectra were recorded on a back illuminated $2048 \times 2048$ pixels CCD camera with a pixel size of $15 \times 15 \mu \mathrm{m}^{2}$. Observed spectra were obtained in échelle

\footnotetext{
${ }^{2}$ http://archive.stsci.edu/kepler/

${ }^{3}$ http://www.tug.tubitak.gov.tr/rtt150_tfosc.php

mode, which provides an effective resolution of $R=\lambda / \Delta \lambda=$ 2500 , which indicates $\Delta \lambda$ value of $2.6 \AA$ around $6500 \AA$ region. The spectra covers usable wavelength range between $3900-9100 \AA$ in 11 orders.

We obtained nine optical spectra of KIC 7385478 between 2014 and 2016 observing seasons. Exposure time of observations are 3200 and $3600 \mathrm{~s}$ depending on atmospheric seeing conditions. Signal-to-noise ratio (SNR) of observed are between 80 and 145, which are estimated via photon statistic. We further obtained high SNR spectra of $\iota$ Psc (HD 222368, F7V, $v_{\mathrm{r}}=5.656 \mathrm{~km} \mathrm{~s}^{-1}$ ), and HD 184499 $\left(\mathrm{G} 0 \mathrm{~V}, v_{\mathrm{r}}=-166.1 \mathrm{~km} \mathrm{~s}^{-1}\right)$ to use as radial velocity template and spectroscopic comparison.

We used standard $\mathrm{IRAF}^{4}$ packages and tasks to reduce all observations. In each observing run, several bias and halogen lamp (flat field) frames were obtained as well as comparison lamp ( $\mathrm{Fe}-\mathrm{Ar}$ ) images, just before or after the target star observation. In the beginning of the reduction process, master bias frame obtained from nightly taken $8-10$ bias frames were subtracted from all object, $\mathrm{Fe}-\mathrm{Ar}$ comparison lamp and halogen lamp frames. Then, normalised master flat field frame was produced from bias corrected halogen lamp frames and all target and $\mathrm{Fe}-\mathrm{Ar}$ spectra were divided by the normalised flat field frame. Cosmic rays removal and scattered light corrections were applied to the bias and flat field corrected images. The spectra from reduced frames were extracted with IRAF task 'apall' under noao.imred.echelle package. $\mathrm{Fe}-\mathrm{Ar}$ images were used for wavelength calibration and finally wavelength calibrated target star spectra were normalised to the unity by using cubic spline functions.

\section{ANALYSIS}

\subsection{Spectral type}

Instead of the published effective temperature estimations in the literature, we use the advantage of having medium resolution spectra of the system to estimate the spectral type and effective temperature, which would be more accurate.

Our preliminary light curve analysis indicates that the secondary component makes $\sim 15 \%$ contribution to the total light of the system, which means the contribution of the secondary component in the spectra taken around secondary minimum would be negligible in our resolution. We have a good spectrum taken at orbital phase $\sim 0.45$ (see Table 1) where the signal of the secondary component fairly diminishes in the resolution of our spectrum, so that we mainly observe the spectrum of the primary component. We adopt this spectrum as reference spectrum for the primary component and compare it with the observed spectra of $\iota$ Psc and HD 184499. The comparison shows that the primary seems hotter than the both standard stars, therefore, we switch to synthetic spectrum fitting method. In general, we use the latest version

\footnotetext{
${ }^{4}$ The Image Reduction and Analysis Facility is hosted by the National Optical Astronomy Observatories in Tucson, Arizona at URL iraf.noao.edu.
} 
Table 1. Log of spectroscopic observations together with measured radial velocities and their corresponding standard errors $(\sigma)$ in $\mathrm{km} \mathrm{s}^{-1}$.

\begin{tabular}{|c|c|c|c|c|c|c|}
\hline \multirow[b]{2}{*}{$\begin{array}{l}\text { HJD } \\
(2400000+)\end{array}$} & \multirow[b]{2}{*}{$\begin{array}{l}\text { Orbital } \\
\text { phase }\end{array}$} & \multirow[b]{2}{*}{$\begin{array}{l}\text { Exposure } \\
\text { time (s) }\end{array}$} & \multicolumn{2}{|c|}{ Primary } & \multicolumn{2}{|c|}{ Secondary } \\
\hline & & & $\mathrm{V}_{\mathrm{r}}$ & $\sigma$ & $\mathrm{V}_{\mathrm{r}}$ & $\sigma$ \\
\hline 56845.4821 & 0.2399 & 3600 & -54.5 & 5.2 & 159.0 & 19.9 \\
\hline 56845.5248 & 0.2657 & 3600 & -46.8 & 7.0 & 158.2 & 23.9 \\
\hline 56846.4677 & 0.8352 & 3600 & 10.2 & 6.3 & -177.1 & 22.5 \\
\hline 56887.4623 & 0.5984 & 3200 & 8.1 & 6.8 & -128.1 & 38.6 \\
\hline 57591.5291 & 0.8948 & 3600 & 11.0 & 10.2 & -136.2 & 41.6 \\
\hline 57600.5477 & 0.3425 & 3600 & -46.8 & 10.9 & 132.6 & 32.4 \\
\hline 57601.3798 & 0.8452 & 3600 & 24.3 & 13.2 & -152.0 & 38.1 \\
\hline 57617.2875 & 0.4543 & 3600 & -27.3 & 4.7 & - & - \\
\hline 57672.3540 & 0.7174 & 3600 & 24.4 & 8.4 & -191.5 & 25.3 \\
\hline
\end{tabular}

of python framework iSpec (Blanco-Cuaresma et al. 2014), which provides easy use of various synthetic spectrum calculation codes. Among these codes, we adopt SPECTRUM ${ }^{5}$ code (Gray \& Corbally 1994) and calculate synthetic spectra by using ATLAS-9 (Castelli \& Kurucz 2004) model atmospheres in conjunction with line list from the third version of the Vienna atomic line database (VALD3) (Ryabchikova et al. 2015). Grids of model atmospheres are taken from the temperature range between 6000 and $7500 \mathrm{~K}$ in steps of $250 \mathrm{~K}$ and gravity $(\log g)$ range between 4.5 and 3.5. In all calculations, we adopt $2 \mathrm{~km} \mathrm{~s}^{-1}$ of microturbulence velocity and solar metallicity. Calculated spectra are convolved with a Gaussian line-spread function to match the resolution of the reference spectrum and this convolution is done either by auxiliary programme smoot $h$ provided by the SPECTRUM code or by built-in function of $i$ Spec code. However, we do not consider the rotational broadening and other broadening mechanisms, which could be ignored due to the relatively large instrumental broadening in our spectra. The models with the temperature $7000 \mathrm{~K}$ and $\log g$ values 4.5 and 4.0 provide very close matches to the reference spectrum. However, calculated physical properties of the system show that the $\log g$ value of the primary component is 4.27 (see Section 3.4). We also use synthetic spectrum fitting routine in $i S p e c$, which mainly adopts method of minimising $\chi^{2}$ value of the difference between the reference spectrum and synthetic spectra. For this purpose, we choose wavelength region of 4 750-5 $700 \AA$ and executed fitting routine by fixing $\log g$ value and choosing different starting parameters for the temperature and metallicity. This method confirmed primary star temperature as $7000 \mathrm{~K}$ and solar metallicity assumption. This temperature corresponds to the $F 1$ spectral type according to the calibration given by Gray (2005). Considering calculated error of the temperature from $\chi^{2}$ minimisation method, temperature steps in ATLAS- 9 grids and current resolution of the observed spectra, uncertainty of the temperature is estimated

\footnotetext{
${ }^{5}$ http://www.appstate.edu/ grayro/spectrum/spectrum.html
}

PASA, 34, e017 (2017)

doi:10.1017/pasa.2017.11


Figure 1. Representation of the observed (black), best matched (red) synthetic spectrum, and residuals (blue) for three regions. Note that we shift the residuals upwards by 0.3 for the sake of simplicity. Panels $a, b$, and $c$ show the regions around $\mathrm{H}_{\beta}$, Mg I triplet, and metallic absorption lines around $5500 \AA$ A, respectively.

to be $150 \mathrm{~K}$. We plot the observed reference spectrum and the best matched synthetic spectrum in Figure 1 for three different regions.

\subsection{Radial velocities and spectroscopic orbit}

We calculate radial velocities of the system by crosscorrelating each observed spectrum with a template spectrum using fxcor task in IRAF (Tonry \& Davis 1979). Here, we adopt $\iota$ Psc as the template since it provides the most similar spectrum to KIC 7385478 and obtained with the same instrumental setup. We consider absorption lines in échelle orders $3,4,5$, and 6, which cover $5000-6800 \AA$, except strongly blended lines and broad lines, such as $\mathrm{H}_{\alpha}$ and $\mathrm{Na}$ I D lines. In Figure 2, we show cross-correlation functions of two spectra obtained around orbital quadratures.

We give $\log$ of observations in Table 1, together with measured radial velocities and their standard errors. Orbital phases in the table are calculated via ephemeris and period given in Borkovits et al. (2016), which we also adopt for 


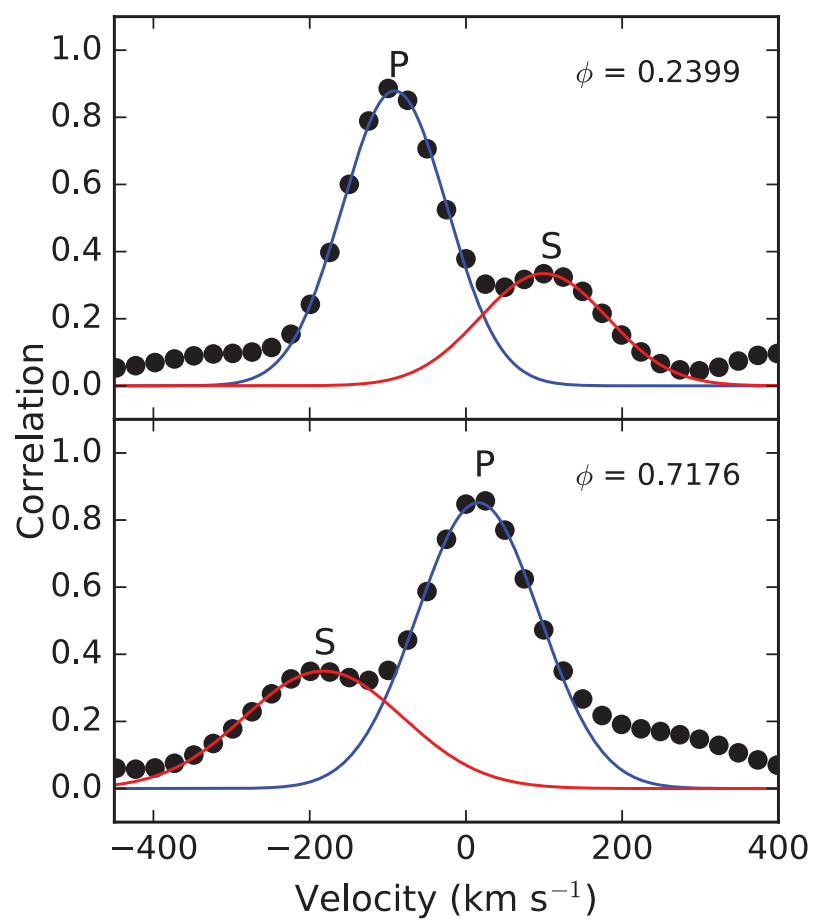

Figure 2. Cross-correlation functions of two spectra obtained in orbital quadratures. The letter $\phi$ shows corresponding orbital phase.

further analysis. Investigating Table 1, one can easily notice the large standard error values for the measured radial velocities of secondary component. This is primarily caused by the small contribution of the secondary component to the total spectrum of the system, which can be evaluated from the cross correlation peaks of components given in Figure 2. We note that $3600 \mathrm{~s}$ of exposure time corresponds to 0.025 orbital phase step and estimated radial velocity shift during this exposure time is about $8 \mathrm{~km} \mathrm{~s}^{-1}$ at the orbital quadratures for the secondary component, and much smaller for the primary component. These shifts are negligible compared to the standard errors of the measured radial velocities; therefore, we can safely ignore the velocity shift due to the long exposure time.

Preliminary light curve examination of KIC 7385478 indicates no evidence for eccentric orbit; therefore, we calculate spectroscopic orbital elements of the system by assuming circular orbit. In addition, we fixed the orbital period and ephemeris to the values given by Borkovits et al. (2016). We use a simple python script written by us, which applies differential corrections by least squares method to all radial velocities, as described in Aitken (1935). We tabulate calculated spectroscopic orbital elements in Table 2 and plot measured radial velocities together with theoretical spectroscopic orbit and residuals from solution in Figure 3. Residuals, which belongs to the primary component scatters over zero level, while the residuals of the secondary component occupy the sub-zero level and this may be interpreted as if the whole fit could be improved further. However, if one consider the
Table 2. Spectroscopic orbital elements of KIC 7385478. $M_{1}$ and $M_{2}$ denote the masses of the primary and secondary component, respectively, while $M$ shows the total mass of the system.

\begin{tabular}{|c|c|}
\hline Parameter & Value \\
\hline$P_{\text {orb }}(\mathrm{d})$ & 1.655473 (fixed) \\
\hline$T_{0}(\mathrm{HJD} 2454+)$ & 954.534784 (fixed) \\
\hline$\gamma\left(\mathrm{km} \mathrm{s}^{-1}\right)$ & $-16.2 \pm 0.8$ \\
\hline$K_{1}\left(\mathrm{~km} \mathrm{~s}^{-1}\right)$ & $38.3 \pm 2.7$ \\
\hline$K_{2}\left(\mathrm{~km} \mathrm{~s}^{-1}\right)$ & $178.8 \pm 2.7$ \\
\hline$e$ & 0 (fixed) \\
\hline$a \sin i\left(\mathrm{R}_{\odot}\right)$ & $7.10 \pm 0.12$ \\
\hline$M \sin ^{3} i\left(\mathrm{M}_{\odot}\right)$ & $1.754 \pm 0.061$ \\
\hline Mass ratio $\left(q=M_{2} / M_{1}\right)$ & $0.21 \pm 0.02$ \\
\hline Fit rms $\left(\mathrm{km} \mathrm{s}^{-1}\right)$ & 6.5 \\
\hline
\end{tabular}

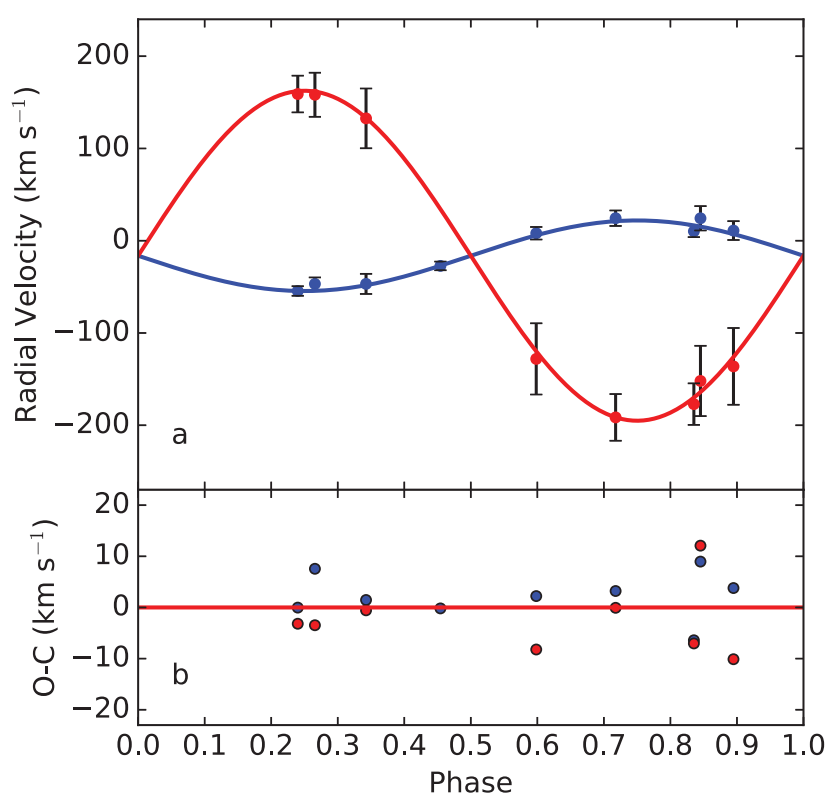

Figure 3. (a) Observed radial velocities of the primary and the secondary (blue and red filled circles, respectively) and their corresponding theoretical representations (blue and red curve). (b) Residuals from theoretical solution.

total rms of the fit given in Table 2 (last row) as one $\sigma$, then the scatter of residuals is inside $\sim 1.5 \sigma$ level and indicates that any further improvement to the orbital solution has no statistical significance, but only cause slight changes in stellar parameters, which would still stay inside corresponding standard error.

\subsection{Light curve modelling}

In case of KIC 7385478, light curve modelling would not be practical by considering 65722 long cadence data points; therefore, we first phase the whole data with respect to the orbital period and then calculate binned light curve with a phase step of 0.002 by using freely available fortran code 


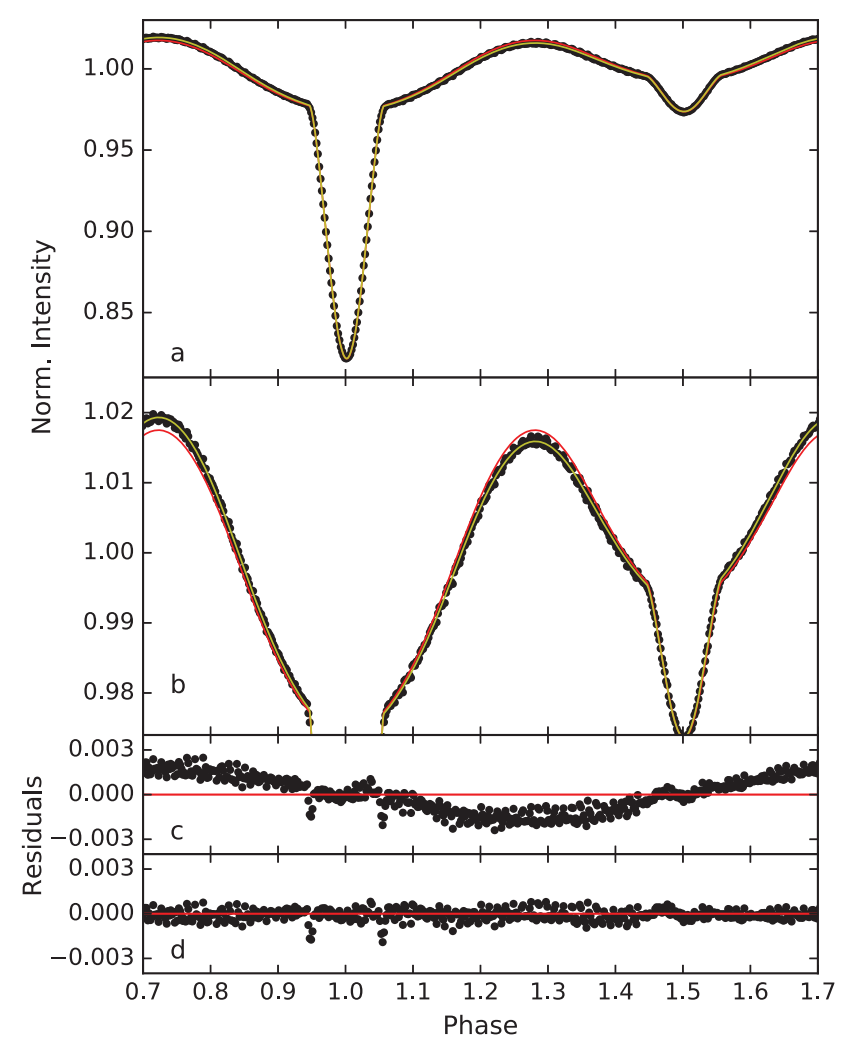

Figure 4. (a) Phase binned light curve of KIC 7385478 (black filled circles) together with best fit models with and without spot (yellow and red curves, respectively). (b) Close up view of the light curve at light maxima. Panel (c) and (d) show residuals from the solutions without and with spot, respectively.

lcbin ${ }^{6}$ written by John Southworth. We use the phase binned light curve for modelling the eclipsing binary. The first step of the light curve modelling is to find geometric and physical parameters of the system, based on phase binned data and the second step is to calculate theoretical light curve with the best geometric and physical parameters and subtract the theoretical model from the whole long cadence data in order to inspect possible out of eclipse variations. We plot the phase binned light curve in Figure 4, panel $a$.

By the quick inspection of the phase binned light curve, one may see that the system is on a circular orbit and there is a possibility of semi-detached configuration for the system. It may also be noticed that light level around 0.75 phase is slightly higher than the light level around 0.25 phase (see Figure 4, panel $b$ ), which means this effect is dominant through 4 yrs of Kepler observations. Difference in the depth of the primary and secondary minima is another important feature of the light curve, which indicates large difference between the temperatures of the components. Shape of the light curve at out of eclipse phases indicates variations beside the geometric eclipse events, such as distortion in geometric shape of the components, reflection, spots, and even oscillations as we will focus on in the next section.

\footnotetext{
${ }^{6} \mathrm{http}: / /$ www.astro.keele.ac.uk/ $\sim \mathrm{jkt} /$ codes.html\#lcbin
}

We use 2015 version of the Wilson-Devinney code (Wilson \& Devinney 1971; Wilson \& Van Hamme 2014) for light curve modelling. Thanks to our spectroscopic observations, we have already determined the two most critical parameters of the light curve modelling process, i.e. effective temperature of the primary component and the mass ratio of the system. We fixed these two parameters during the modelling. Since the effective temperature of the primary component is at critical location where the convective outer envelope is very thin or almost becomes radiative, we carried out solutions by setting gravity darkening $\left(g_{1}\right)$ and albedo $\left(A_{1}\right)$ values to 0.32 and 0.5 , respectively (for convective envelopes) and setting both parameters to 1.0 (for radiative envelopes). In both cases, there is almost negligible difference between solutions with radiative envelope and convective envelope assumption, where convective envelope assumption leads to a slightly lower residuals; thus, we continue with 0.32 and 0.5 values for $g_{1}$ and $A_{1}$, respectively. We adopt $g_{2}$ and $A_{2}$ values as 0.32 and 0.5 for the secondary component, which are typical for convective envelopes. Both light curve and spectroscopic orbit solution indicate circular orbit for the system; therefore, we assume synchronous rotation for the components, which is proper for circular orbits, and fix rotation parameter $(F)$ of each component to 1.0. Here, $F$ is defined as the ratio of the axial rotation rate to the orbital rate. Linear limb darkening coefficients $\left(x_{1}, x_{2}\right)$ of the components are adopted from van Hamme (1993).

We start analysis with detached configuration, therefore, we consider inclination of the orbit $(i)$, temperature of the secondary component $\left(T_{2}\right)$, dimensionless omega potentials of the primary and the secondary component $\left(\Omega_{1}, \Omega_{2}\right)$, luminosity of the primary component $\left(L_{1}\right)$ as adjustable parameters. In addition, we observe a general phase shift of 0.002 in the phase binned light curve, which possibly arises from a shift in the adopted ephemeris due to the third body (Borkovits et al. 2016); hence, we also adjust phase shift during analysis. In a few iterations, we observe that the $\Omega_{2}$ value jiggles around the inner critical potential value and secondary component has entirely filled its Roche-lobe in the corresponding Roche geometry. Then we switch to the semi-detached configuration and fixed $\Omega_{2}$. In this case, we consider distorted shape of the secondary component and adopt $g_{2}, A_{2}$, and $x_{2}$ as adjustable parameters during iterations. After a few iterations, we achieve statistically the best parameter set, however, we still observe that the residuals from the solution exhibit additional wave-like variation through an orbital cycle.

During the iterations, we noticed that the contribution of the secondary component to the total light is not more than $15 \%$; therefore, we expect negligible contribution from the secondary component to the wave-like variation in residuals. The most possible explanation could be that the Rochelobe filled secondary star transfers its own mass to the primary component through the inner Lagrange point, $L 1$, of the system. The transferred mass possibly hits directly to the photosphere of the primary component without forming an accretion disk, thus forms a local region warmer than the 
Table 3. Light curve modelling results of KIC 7385478. $\left\langle r_{1}\right\rangle$ and $\left\langle r_{2}\right\rangle$ denote mean fractional radii of the primary and the secondary components, respectively. Spot parameters are given in the order of co-latitude $(\theta)$, longitude $(\varphi)$, radius $\left(r_{\text {spot }}\right)$, and temperature factor (TF). Internal errors of the adjusted parameters are given in parentheses for the last digits. Asterix symbols in the table denote fixed value for the corresponding parameters.

\begin{tabular}{lc}
\hline \hline Parameter & Value \\
\hline$q$ & $0.21^{*}$ \\
$T_{1}(\mathrm{~K})$ & $7000^{*}$ \\
$g_{1}, g_{2}$ & $0.32^{*}, 0.270(5)$ \\
$A_{1}, A_{2}$ & $0.5^{*}, 0.699(3)$ \\
$F_{1}=F_{2}$ & $1.0^{*}$ \\
Phase shift & $0.00160(2)$ \\
$i\left(^{\circ}\right)$ & $70.966(8)$ \\
$T_{2}(\mathrm{~K})$ & $4293(150)$ \\
$\Omega_{1}$ & $4.9582(43)$ \\
$\Omega_{2}$ & $2.2574^{*}$ \\
$L_{1} /\left(L_{1}+L_{2}\right)$ & $0.8590(6)$ \\
$x_{1} b o l, x_{2} b o l$ & $0.471^{*}, 0.531^{*}$ \\
$x_{1}, x_{2}$ & $0.462^{*}, 0.344(8)$ \\
$\left\langle r_{1}\right\rangle,\left\langle r_{2}\right\rangle$ & $0.2114(2), 0.2527^{*}$ \\
$\theta\left(^{\circ}\right)$ & $98(6)$ \\
$\varphi\left({ }^{\circ}\right)$ & $93(1)$ \\
$r_{\text {spot }}\left({ }^{\circ}\right)$ & $13(1)$ \\
$\mathrm{TF}$ & $1.022(2)$ \\
Model rms & $1.1 \times 10^{-4}$ \\
\hline \hline
\end{tabular}

surrounding photosphere. Therefore, we consider this hot region as a bright spot on the primary component and add a single bright spot into our light curve model. At first step, we adopt spot longitude and radius as adjustable parameters together with eclipsing binary parameters and fixed the spot co-latitude and temperature factor to $45^{\circ}$ and 1.03 , respectively. After these parameters are adjusted, we fix the spot longitude and radius, and adjust spot co-latitude and temperature factor. Until we achieve the best solution, we adjust different number of spot parameters simultaneously with a different combinations. When we reach the best solution, we adopt all parameters (spot and eclipsing binary) adjustable and run a single iteration in order to obtain statistical uncertainties. We tabulate our results in Table 3. Note that we do not give the internal error of the $T_{2}$ since it is unrealistically small $(\sim 1 \mathrm{~K})$; therefore, we adopt the uncertainty of $T_{1}$ estimated in Section 3.1 as the uncertainty of $T_{2}$. In Figure 4, we over plot the best fit models of spotless and spotted solutions (panel $a$ and $b$ ) and show residuals from both solutions in panel $c$ and $d$ in the same figure.

\subsection{Physical properties and evolutionary status}

Combining results from spectroscopic orbital solution and light curve modelling, we calculate absolute physical parameters of the system listed in Table 4. Inspecting the parameters, we immediately see that the secondary component has a very large radius compared to its mass which causes lower grav-
Table 4. Absolute physical properties of KIC 7385478. Error of each parameter is given in parenthesis for the last digits.

\begin{tabular}{lcl}
\hline \hline Parameter & Primary & Secondary \\
\hline Spectral Type & F1V & K4 III-IV \\
Mass $\left(M_{\odot}\right)$ & $1.71(8)$ & $0.37(4)$ \\
Radius $\left(\mathrm{R}_{\odot}\right)$ & $1.59(3)$ & $1.90(3)$ \\
$\log L / \mathrm{L}_{\odot}$ & $0.737(52)$ & $0.043(82)$ \\
$\log g(\mathrm{cgs})$ & $4.269(7)$ & $3.444(31)$ \\
$M_{\text {bol }}(\mathrm{mag})$ & $2.91(13)$ & $4.64(21)$ \\
\hline \hline
\end{tabular}

ity compared to a typical main sequence star. A typical main sequence star has a radius of $\sim 0.41 \mathrm{R}_{\odot}($ Gray 2005). Adopting temperature calibration of Gray (2005), we estimate the spectral type of the secondary as K4 III-IV. All these indicate that the lower mass secondary component has already evolved off the main sequence, which seems contradictory to the stellar evolution theory. However, we know that the secondary component has already filled its Roche-lobe and there must be mass transfer from secondary to the primary via inner Lagrange point, $L 1$. The situation could be explained as the secondary component was actually the more massive component in the system and as it evolved off the main sequence, it filled its Roche-lobe and started a mass transfer to the less massive component (in our case, the primary component). We observe the effect of the mass transfer as hot spot on the primary component. Continuous mass transfer in time finally reversed the mass ratio of the system; therefore, the final configuration became like a more massive main sequence star and a less massive sub-giant star. This is typical scenario adopted for Algols, which are well known to have reverted their mass ratio because of Roche-lobe overflow.

In Figure 5, we show the position of the primary component of KIC 7385478 on Hertzsprung-Russel diagram, together with some eclipsing binaries with known $\gamma$ Doradus type pulsating components, i.e. VZ CVn (Ibanoğlu et al. 2007), CoRot 102918586 (Maceroni et al. 2013), KIC 11285625 (Debosscher et al. 2013), KIC 9851142 (Çakırlı 2015), KIC 9851944 (Guo et al. 2016), V2653 Oph (Çakırlı \& Ibanoglu 2016), CoRot 100866999 (Chapellier \& Mathias 2013). Our target is located in the middle of the $\gamma$ Doradus instability strip given by Warner et al. (2003), which takes us to the possibility of $\gamma$ Doradus type pulsation on the primary component.

\subsection{The out-of-eclipse variations}

In order to inspect the out-of-eclipse variations, we first construct a theoretical light curve by using the parameters in Table 3, then subtract it from whole long cadence data and obtain residuals. In this process, we first divide the whole long cadence data into subsets where each subset covers a single orbital cycle. Then, we run differential correction programme of the Wilson-Devinney code by only adjusting ephemeris 


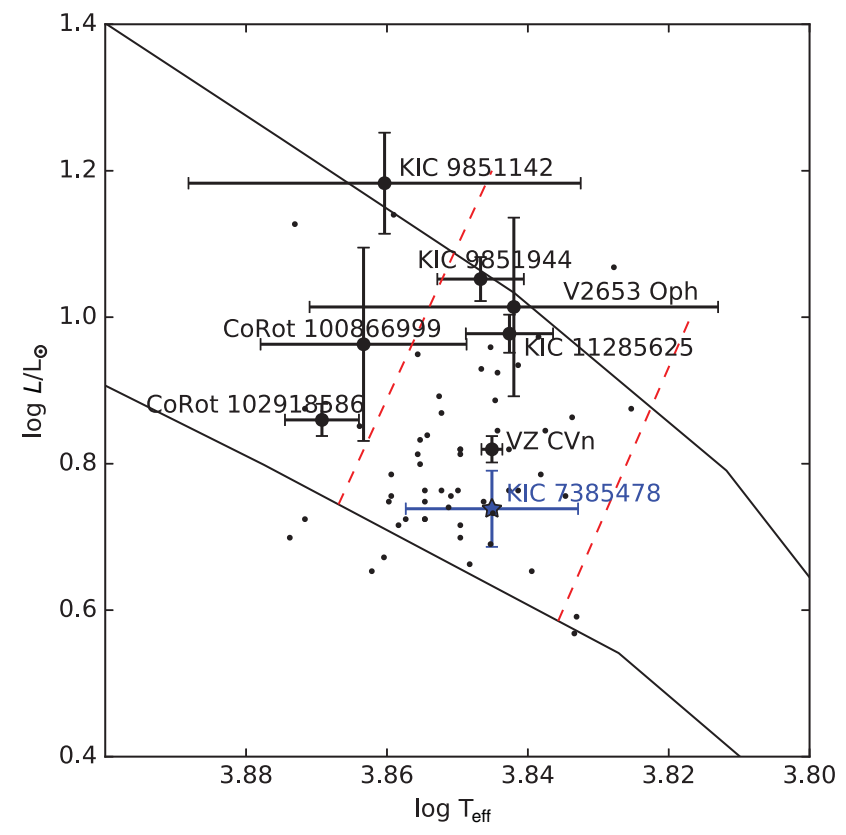

Figure 5. Position of the primary component of KIC 7385478 on Hertzsprung-Russel diagram (star symbol in blue). Black dots show confirmed $\gamma$ Doradus stars from Henry, Fekel, \& Henry (2005), filled large black circles denote discovered pulsating components in eclipsing binaries. Red dashed lines indicate theoretical cool and hot boundary of $\gamma$ Doradus instability strip (Warner, Kaye, \& Guzik 2003). Black continuous curves show zero age and terminal age main sequences, taken from Pols et al. (1998).

reference time of the related subset and keeping all remaining parameters fixed. This process eliminates any shift in the ephemeris reference time due to the light time travel effect caused by the third body and gives correct residuals.

In Figure 6, we plot the whole residuals (panel $a$ ), and a sample of residuals covering a time span of a month (panel $b$ and $c$ ). One may easily notice that removing eclipsing binary model from the data unravels a clear variation with a dominant period of $\sim 0.5 \mathrm{~d}$ (panel $b$ ) with variable amplitude, which suggests the possibility of $\gamma$ Doradus type pulsation (Kaye et al. 1999) on the primary component, with a beat period of about $12 \mathrm{~d}$. In addition, we observe occasional increase in amplitude of residuals (panel $c$ ), where the most dominant period becomes the orbital period, however, by keeping 0.5 $\mathrm{d}$ variation as small humps and pits through an orbital cycle.

We apply multi-frequency analysis to the residual data using pysca software package (Herzberg \& Glogowski 2014) to investigate these variations. In case of continuous and long time series photometry, pysca is very practical to automatically extract significant frequencies above a defined SNR limit. We start with Fourier analysis of the data for the frequency between 0 and 24.498 cycle/d (c/d), where the 24.498 denotes the nyquist frequency. In Figure 7, we show amplitude spectrum of the residuals ( $\operatorname{panel} a$ ). We observe that the dominant frequencies are located in lower frequency region $(f<5 \mathrm{c} / \mathrm{d})$ and consider this region for frequency extraction process. In this process, the most dominant frequency in the amplitude spectrum is determined. Then its amplitude and phase are calculated via $A_{\mathrm{i}} \Sigma \sin \left[2 \pi\left(f_{\mathrm{i}} t+\phi_{i}\right)\right]$, where $t$ is the time of the corresponding measurement, while $A_{\mathrm{i}}, f_{\mathrm{i}}$, and $\phi_{\mathrm{i}}$ show the amplitude, frequency, and phase of the $i$ th frequency, respectively. Next, this frequency is removed from the data and the same process is repeated for the remaining 'prewhitened' residuals. We adopt criteria of Breger et al. (1993), which puts a lower SNR limit of 4 for a frequency to be accepted as significant. Uncertainties of the extracted frequencies are estimated as $\sim 7 \times 10^{-3} \mathrm{~d}^{-1}$, which is determined by using the Rayleigh criterion.

This process leads to 735 frequencies above SNR limit of 4 . The most dominant two peaks are located at 2.0252 and $1.9427 \mathrm{c} / \mathrm{d}$, corresponding 0.4938 and $0.5147 \mathrm{~d}$ and we define these frequencies as $\gamma$ Doradus type pulsation frequencies. Third and fourth peaks are 0.6034 and $0.6023 \mathrm{c} / \mathrm{d}$, corresponding 1.6573 and $1.6603 \mathrm{~d}$, and these frequencies indicate the orbital frequency. Beyond the first four frequencies, we do not find any independent frequency but low- and high-order combination of pulsation and orbital frequencies. We list the extracted frequencies in Table A1.

In Figure 7, we also show close view around orbital frequency (panel $b$ ) and pulsation frequencies (panel $c$ and $d$ ). One may easily notice that peaks at pulsation frequencies are single and sharp peaks, while the peak at the orbital frequency is more shallow in amplitude and broader. This picture leads us to calculate amplitude spectrum of long cadence data for each Kepler data quarter separately and investigate behaviour of the dominant peaks in 4 yrs time range. We plot amplitude spectrum of each quarter separately in Figure 8. Amplitudes of the pulsation frequencies are almost stable in all quarters, while the amplitude of the orbital frequency clearly varies in time, which causes shallow and broader peak(s) at the orbital frequency in the amplitude spectrum of the $4 \mathrm{yr}$ long cadence data.

\section{SUMMARY AND DISCUSSION}

Photometric and spectroscopic analysis of KIC 7385478 shows that the system is a low mass ratio $(q=0.21)$ eclipsing binary formed by an F1V primary, and a K4III-IV secondary components which entirely fills its Roche-lobe. This means the system can be classified as semi-detached. Physical properties of the components suggest that the low mass secondary component has already evolved off the main sequence before the dwarf primary component, which is not expected in the scope of the stellar evolution theory. Continuous mass transfer between the components could explain this situation, which could change the roles (and masses) of the components in long time scales. If the mass transfer does not lead to a disk, a hot spot forms on the photosphere of the mass gaining component, where the streaming matter directly hits to its photosphere. Almost continuous Kepler long cadence photometry through 4 yrs indicates a brightness level difference between orbital quadrature phases (i.e. 0.25 and 0.75 phases), which could be explained by this kind of a hot spot on the primary (mass gaining) component. At that 


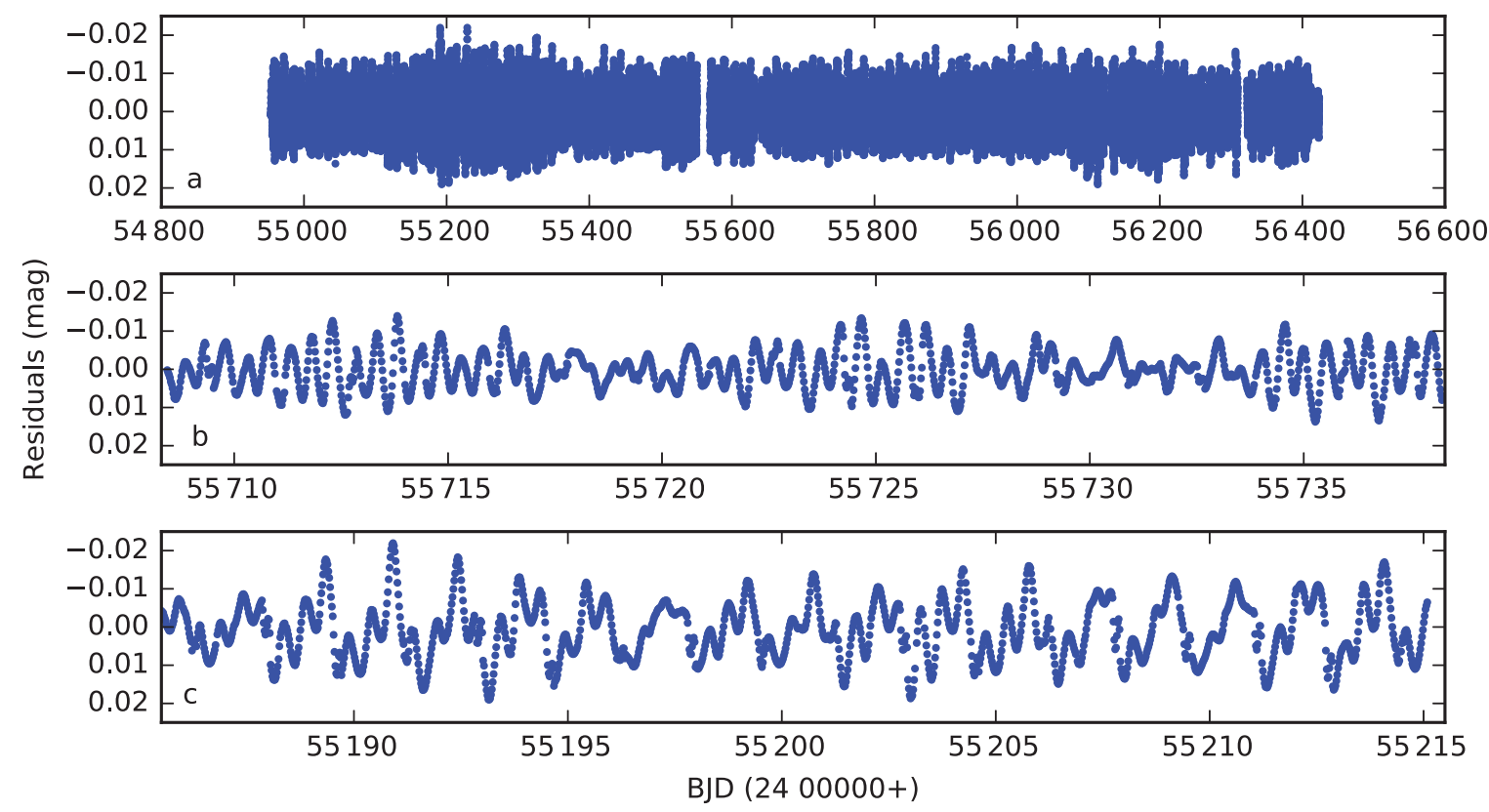

Figure 6. (a) Residuals from whole long cadence data. (b) Portion of residuals around BJD $=2455723$, where the variation amplitude is smaller. (c) Residuals around BJD $=2455$ 200, where the variation amplitude is larger.


Figure 7. (a) The whole amplitude spectrum of the residuals. (b) Close view of the frequency range where the orbital frequency is located. Panel $c$ and $d$ are similar to the panel $b$ but for pulsation frequencies.

point, the assumption of an impact system (no disc) can be justified by the mass ratio $(0.22)$ and fractional radius of the primary (0.21) according to Lubow-Shu criterium (Lubow $\&$ Shu 1975) for disc formation. Furthermore, we do not observe any emission feature in $\mathrm{H}_{\alpha}$, indicates the absence of the disk and this is consistent with impact system assumption.

The position of the primary component on HR diagram corresponds to the middle of the region where $\gamma$ Doradus variables are located and suggests intrinsic light variations related to pulsation. When we subtract the eclipsing binary model from the long cadence data, we see clear signal which has a dominant period of $\sim 0.5 \mathrm{~d}$ indicating $\gamma$ Doradus type pulsations and confirms the intrinsic variation suggested by the position on HR diagram. However, we also observe occasional increase in the amplitude of the residuals where the dominant period becomes the orbital period, including 


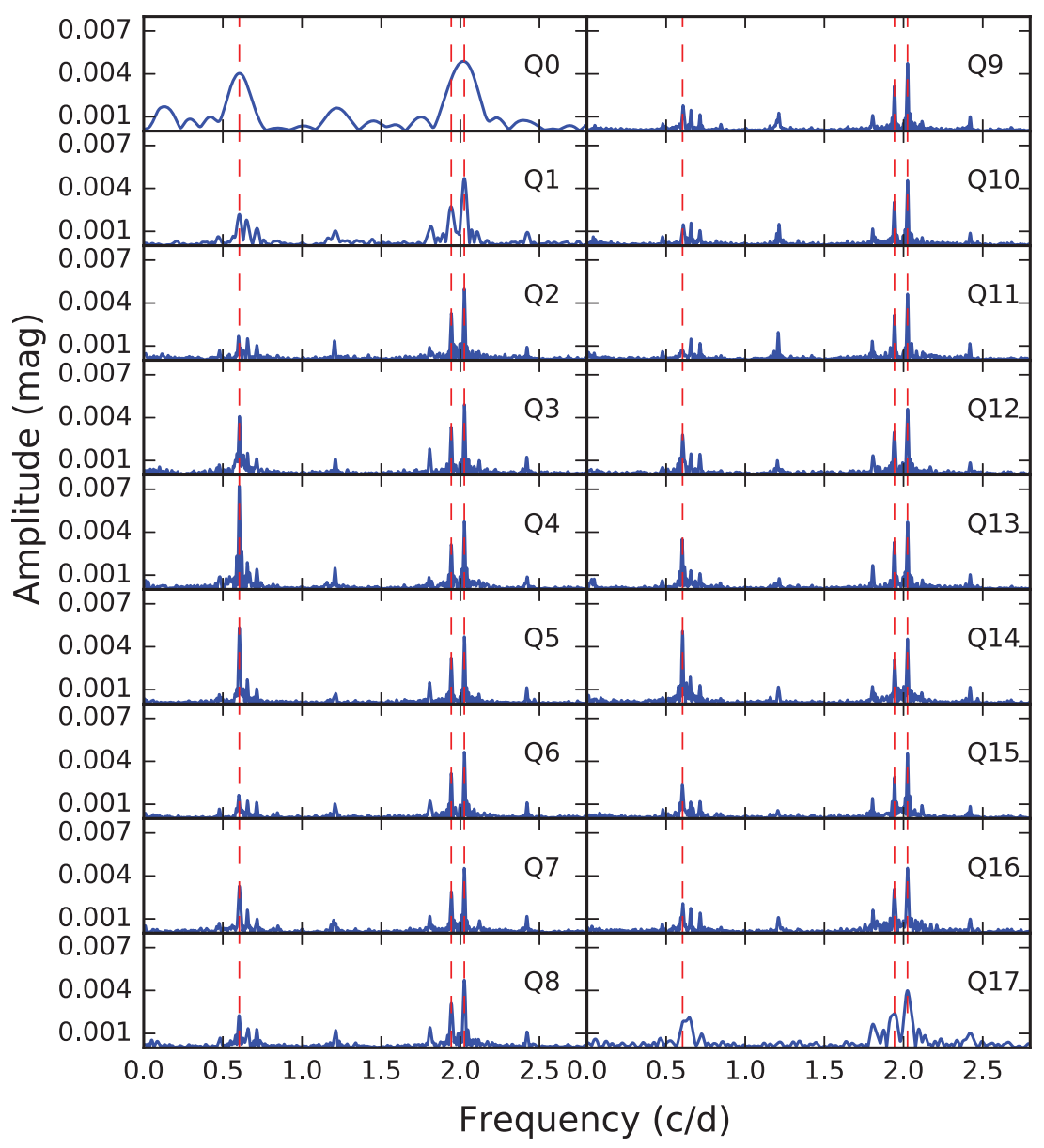

Figure 8. Amplitude spectrum of each quarter. We mark the location of the orbital frequency and the most dominant two frequencies (i.e. pulsation frequencies) with red vertical dashed lines and label each plot window according to its quarter number. Note that Q0 and Q17 contain less number of data points compared to the other quarters, thus peaks of dominant frequencies are broad in their amplitude spectrum.

$\sim 0.5 \mathrm{~d}$ variation as a smaller amplitude variation. Multiple frequency analysis of the residuals results in 735 frequencies, where the first two frequencies indicate $\gamma$ Doradus type pulsations, while the third and the fourth frequencies correspond to the orbital frequency. Most of the remaining 731 frequencies correspond to either the orbital frequency or its harmonics. This indicates an additional light variation with a period almost identical to the orbital one and this variation is usually suppressed by the light variation originating from pulsations.

Figures 7 and 8 clearly show that the amplitude of the orbital frequency varies in time and this causes broad and shallow peak structure at the orbital frequency in amplitude spectrum of $4 \mathrm{yr}$ data, which is another evidence for the additional light variation mentioned above. Possible star spot activity originating from the cool secondary component could easily cause this kind variation, thus leads to many low- and high-order combinations of the pulsation and orbital frequencies in the amplitude spectrum, especially in period analysis of continuous long-term data, which covers $\sim 4$ yrs in case of KIC 7385478. It is also known that stellar magnetic activity may cause orbital period modulations via mechanism proposed by Applegate (1992). Another possibility for orbital period modulations is variable mass transfer rate in the donor. Since the typical time scales for Applegate mechanism and variable mass transfer rate are decades or longer, these are not comparable to the modulations observed in residual data of KIC 7385478, therefore are not likely.

Estimated spectral type and luminosity class of the secondary component provide support for the spot activity possibility. According to the eclipsing binary model, $14 \%$ contribution of the secondary component to the total light is expected at very broad band Kepler filter, which means we may observe small amplitude light variation due to the possible spot activity of the secondary star. However, when we check the observed spectra of the system, we do not observe any emission feature in $\mathrm{Ca}$ II $\mathrm{H} \& \mathrm{~K}$ lines, which are very sensitive to the chromospheric activity in cool stars. Considering the temperatures and radii of the components, one may easily conclude that the contribution of the 
secondary component to the total light around $3950 \AA$ is almost completely negligible, hence, considering only the spectroscopic indicators, we may not arrive at the conclusion on the existence of star spot activity in case of KIC 7385478. Therefore, we can still speculate that the secondary component might have star spot activity, which seems the most possible cause of the occasional amplitude increase in the light residuals.

\section{ACKNOWLEDGEMENTS}

We thank to TUBITAK for a partial support in using RTT150 (Russian-Turkish 1.5-m telescope in Antalya) with project number 14BRTT150-667. This paper includes data collected by the Kepler mission. Funding for the Kepler mission is provided by the NASA Science Mission Directorate. Some of the data presented in this paper were obtained from the Mikulski Archive for Space Telescopes (MAST). STScI is operated by the Association of Universities for Research in Astronomy, Inc., under NASA contract NAS5-26555. Support for MAST for non-HST data is provided by the NASA Office of Space Science via grant NNX13AC07G and by other grants and contracts.

\section{REFERENCES}

Aerts, C., Christensen-Dalsgaard, J., \& Kurtz, D. W. 2010, Asteroseismology, ed. W. B. Burton and V. Trimble (New York: Springer), 49

Aitken, R. G. 1935, The binary stars (2nd edn.; New York and London: McGraw-Hill)

Applegate, J. H. 1992, ApJ, 385, 621

Armstrong, D. J., Gómez Maqueo Chew, Y., Faedi, F., \& Pollacco, D. 2014, MNRAS, 437, 3473

Blanco-Cuaresma, S., Soubiran, C., Heiter, U., \& Jofré, P. 2014, A\&A, 569, A111

Borkovits, T., et al. 2016, MNRAS, 455, 4136

Breger, M., et al. 1993, A\&A, 271, 482

Çakırlı, Ö. 2015, NewA, 38, 55

Çakırlı, Ö., \& Ibanoglu, C. 2016, NewA, 45, 36

Castelli, F., \& Kurucz, R. L. 2004, in Proc. IAU Symp. 210, Modelling of Stellar Atmospheres, Piskunov, N., Weiss, W. W., \& Gray, D. F., eds. (San Francisco: ASP), A20, arXiv:astro$\mathrm{ph} / 0405087$

Chapellier, E., \& Mathias, P. 2013, A\&A, 556, A87

Cunha, M. S., et al. 2007, A\&ARev, 14, 217

Debosscher, J., et al. 2013, A\&A, 556, A56

Gray, D. F. 2005, The Observation and Analysis of Stellar Photospheres (3rd edn.; Cambridge: Cambridge University Press)

Gray, R. O., \& Corbally, C. J. 1994, AJ, 107, 742

Guo, Z., Gies, D. R., Matson, R. A., \& García Hernández, A. 2016, ApJ, 826, 69

Henry, G. W., Fekel, F. C., \& Henry, S. M. 2005, AJ, 129, 2815

Herzberg, W., \& Glogowski, K. 2014, Precision Asteroseismology, 301,421

Ibanoğlu, C., Taş, G., Sipahi, E., \& Evren, S. 2007, MNRAS, 376, 573

Kaye, A. B., Handler, G., Krisciunas, K., Poretti, E., \& Zerbi, F. M. 1999, PASP, 111, 840

Lubow, S. H., \& Shu, F. H. 1975, ApJ, 198, 383
Maceroni, C., Montalbán, J., Gandolfi, D., Pavlovski, K., \& Rainer, M. 2013, A\&A, 552, A60

Pigulski, A., Pojmański, G., Pilecki, B., \& Szczygieł, D. M. 2009, AcAau, 59, 33

Pinsonneault, M. H., et al. 2012, ApJS, 199, 30

Pols, O. R., Schröder, K.-P., Hurley, J. R., Tout, C. A., \& Eggleton, P. P. 1998, MNRAS, 298, 525

Prša, A., et al. 2011, AJ, 141, 83

Ryabchikova, et al. 2015, PhyS, 90, 054005

Slawson, R. W., et al. 2011, AJ, 142, 160

Tonry, J., \& Davis, M. 1979, AJ, 84, 1511

Uytterhoeven, K., et al. 2011, A\&A, 534A, 125

van Hamme, W. 1993, AJ, 106, 2096

Warner, P. B., Kaye, A. B., \& Guzik, J. A. 2003, ApJ, 593, 1049

Wilson, R. E., \& Devinney, E. J. 1971, ApJ, 166, 605

Wilson, R. E., \& Van Hamme, W. 2014, ApJ, 780, 151

\section{A Multi-frequency analysis results}

Table A1. Extracted frequencies in multi-frequency analysis. N, $\mathrm{F}, \mathrm{A}, \mathrm{P}$, and SNR means number, frequency (in $c / d$ ), amplitude (in mmag), phase, and signal-to-noise ratio, respectively.

\begin{tabular}{lcccc}
\hline \hline$N$ & $F$ & $A$ & $P$ & SNR \\
\hline 1 & 2.0252 & 0.470 & 0.2618 & 275.8 \\
2 & 1.9428 & 0.315 & 0.6111 & 210.5 \\
3 & 0.6035 & 0.183 & 0.7206 & 105.5 \\
4 & 0.6023 & 0.169 & 0.5600 & 84.7 \\
5 & 0.6563 & 0.143 & 0.5643 & 76.2 \\
6 & 0.6055 & 0.127 & 0.1400 & 71.5 \\
7 & 1.8052 & 0.124 & 0.0554 & 91.7 \\
8 & 0.7138 & 0.115 & 0.7896 & 69.5 \\
9 & 2.4208 & 0.102 & 0.4827 & 87.2 \\
10 & 0.6048 & 0.090 & 0.3848 & 62.7 \\
11 & 0.6062 & 0.079 & 0.5564 & 50.9 \\
12 & 1.2087 & 0.058 & 0.1224 & 50.2 \\
13 & 1.2119 & 0.064 & 0.5436 & 48.1 \\
14 & 0.6008 & 0.065 & 0.7873 & 42.2 \\
15 & 0.4780 & 0.061 & 0.4596 & 42.2 \\
16 & 2.1178 & 0.060 & 0.4606 & 54.5 \\
17 & 3.7537 & 0.058 & 0.3023 & 67.9 \\
18 & 0.6110 & 0.062 & 0.5936 & 38.9 \\
19 & 0.6002 & 0.054 & 0.2745 & 39.7 \\
20 & 0.6084 & 0.045 & 0.1297 & 38.7 \\
21 & 0.6028 & 0.050 & 0.7349 & 36.8 \\
22 & 1.2041 & 0.051 & 0.3854 & 35.8 \\
23 & 0.5983 & 0.034 & 0.3999 & 29.4 \\
24 & 1.2051 & 0.036 & 0.5672 & 32.1 \\
25 & 0.8446 & 0.036 & 0.8880 & 27.6 \\
26 & 1.2110 & 0.036 & 0.6077 & 28.4 \\
27 & 1.8109 & 0.033 & 0.6449 & 29.9 \\
28 & 0.6041 & 0.035 & 0.0754 & 25.2 \\
29 & 0.6075 & 0.034 & 0.5545 & 26.5 \\
30 & 1.2099 & 0.032 & 0.5354 & 27.3 \\
31 & 0.5956 & 0.030 & 0.6500 & 22.3 \\
32 & 1.1569 & 0.026 & 0.9567 & 23.0 \\
33 & 0.5993 & 0.030 & 0.3687 & 23.2 \\
34 & 1.6469 & 0.024 & 0.7514 & 23.8 \\
35 & 0.5918 & 0.025 & 0.1310 & 19.6 \\
36 & 0.5970 & 0.021 & 0.9181 & 20.0 \\
37 & 0.0008 & 0.024 & 0.8344 & 18.6 \\
\hline \hline & & & &
\end{tabular}


Table A1. Continued.

\begin{tabular}{|c|c|c|c|c|}
\hline$N$ & $F$ & $A$ & $P$ & SNR \\
\hline 38 & 1.4211 & 0.022 & 0.8007 & 20.5 \\
\hline 39 & 2.8693 & 0.022 & 0.3247 & 24.7 \\
\hline 40 & 2.6815 & 0.022 & 0.0867 & 23.9 \\
\hline 41 & 0.6091 & 0.026 & 0.5645 & 18.4 \\
\hline 42 & 0.6101 & 0.022 & 0.8878 & 20.5 \\
\hline 43 & 1.2078 & 0.020 & 0.8653 & 20.3 \\
\hline 44 & 0.0129 & 0.021 & 0.2401 & 15.2 \\
\hline 45 & 1.8117 & 0.020 & 0.2413 & 20.2 \\
\hline 46 & 1.3322 & 0.019 & 0.7038 & 18.6 \\
\hline 47 & 0.6197 & 0.021 & 0.8756 & 16.6 \\
\hline 48 & 3.8374 & 0.020 & 0.9090 & 25.4 \\
\hline 49 & 1.1790 & 0.018 & 0.4249 & 17.4 \\
\hline 50 & 0.6662 & 0.017 & 0.4237 & 15.9 \\
\hline 51 & 3.0771 & 0.019 & 0.1901 & 22.3 \\
\hline 52 & 0.5902 & 0.016 & 0.0661 & 15.7 \\
\hline 53 & 0.6144 & 0.017 & 0.9196 & 15.4 \\
\hline 54 & 0.6162 & 0.016 & 0.5804 & 15.6 \\
\hline 55 & 1.2030 & 0.018 & 0.0640 & 17.3 \\
\hline 56 & 0.0441 & 0.018 & 0.9902 & 13.9 \\
\hline 57 & 0.0103 & 0.025 & 0.7827 & 13.7 \\
\hline 58 & 2.4687 & 0.018 & 0.5315 & 18.9 \\
\hline 59 & 4.4414 & 0.017 & 0.7160 & 24.4 \\
\hline 60 & 0.8171 & 0.018 & 0.0733 & 15.3 \\
\hline 61 & 2.7390 & 0.018 & 0.4742 & 19.9 \\
\hline 62 & 0.6216 & 0.018 & 0.4458 & 15.1 \\
\hline 63 & 1.2849 & 0.017 & 0.1149 & 16.3 \\
\hline 64 & 4.6194 & 0.016 & 0.5229 & 23.7 \\
\hline 65 & 2.3371 & 0.016 & 0.3393 & 17.2 \\
\hline 66 & 1.1995 & 0.018 & 0.3142 & 14.7 \\
\hline 67 & 1.2005 & 0.018 & 0.6592 & 17.3 \\
\hline 68 & 2.6293 & 0.014 & 0.3300 & 17.5 \\
\hline 69 & 0.4964 & 0.013 & 0.1267 & 13.2 \\
\hline 70 & 4.8330 & 0.013 & 0.6804 & 22.5 \\
\hline 71 & 3.0200 & 0.014 & 0.3884 & 18.4 \\
\hline 72 & 0.0309 & 0.014 & 0.8959 & 11.9 \\
\hline 73 & 1.2133 & 0.014 & 0.6766 & 14.5 \\
\hline 74 & 0.6301 & 0.011 & 0.8103 & 13.1 \\
\hline 75 & 3.1345 & 0.014 & 0.0059 & 18.4 \\
\hline 76 & 0.0524 & 0.017 & 0.4613 & 11.4 \\
\hline 77 & 3.6250 & 0.010 & 0.0494 & 19.6 \\
\hline 78 & 1.8136 & 0.013 & 0.6465 & 15.2 \\
\hline 79 & 0.6280 & 0.014 & 0.5003 & 12.7 \\
\hline 80 & 0.6128 & 0.014 & 0.0515 & 13.1 \\
\hline 81 & 1.5403 & 0.014 & 0.3901 & 14.6 \\
\hline 82 & 4.0154 & 0.014 & 0.2136 & 20.2 \\
\hline 83 & 2.5991 & 0.014 & 0.2656 & 16.6 \\
\hline 84 & 0.6177 & 0.014 & 0.2119 & 12.7 \\
\hline 85 & 3.4113 & 0.013 & 0.2584 & 18.7 \\
\hline 86 & 0.0019 & 0.015 & 0.5863 & 11.6 \\
\hline 87 & 0.1168 & 0.012 & 0.4075 & 11.0 \\
\hline 88 & 3.2333 & 0.013 & 0.5021 & 18.2 \\
\hline 89 & 2.6566 & 0.014 & 0.8287 & 16.6 \\
\hline 90 & 1.3387 & 0.013 & 0.1568 & 14.1 \\
\hline 91 & 1.4590 & 0.013 & 0.0607 & 14.5 \\
\hline 92 & 1.2062 & 0.015 & 0.4634 & 14.7 \\
\hline 93 & 0.5925 & 0.011 & 0.3193 & 12.6 \\
\hline 94 & 1.7284 & 0.013 & 0.1816 & 14.9 \\
\hline 95 & 0.0826 & 0.009 & 0.3652 & 10.7 \\
\hline 96 & 0.6016 & 0.011 & 0.1482 & 12.8 \\
\hline 97 & 0.5811 & 0.013 & 0.7875 & 12.2 \\
\hline 98 & 2.1706 & 0.012 & 0.0829 & 15.3 \\
\hline 99 & 1.2105 & 0.013 & 0.4646 & 16.1 \\
\hline
\end{tabular}

Table A1. Continued.

\begin{tabular}{|c|c|c|c|c|}
\hline$N$ & $F$ & A & $P$ & SNR \\
\hline 100 & 2.4168 & 0.011 & 0.4929 & 15.5 \\
\hline 101 & 1.8152 & 0.016 & 0.2536 & 14.6 \\
\hline 102 & 1.2604 & 0.012 & 0.3536 & 13.4 \\
\hline 103 & 0.0039 & 0.010 & 0.0208 & 10.5 \\
\hline 104 & 2.2210 & 0.013 & 0.3379 & 15.1 \\
\hline 105 & 1.2201 & 0.011 & 0.5429 & 13.3 \\
\hline 106 & 2.8073 & 0.012 & 0.3263 & 16.0 \\
\hline 107 & 0.7347 & 0.012 & 0.5311 & 12.2 \\
\hline 108 & 0.0472 & 0.008 & 0.4934 & 10.1 \\
\hline 109 & 4.2289 & 0.010 & 0.0992 & 19.0 \\
\hline 110 & 0.2200 & 0.010 & 0.1354 & 10.8 \\
\hline 111 & 0.0095 & 0.014 & 0.4078 & 10.5 \\
\hline 112 & 3.7550 & 0.012 & 0.4206 & 17.9 \\
\hline 113 & 0.5854 & 0.014 & 0.8983 & 11.5 \\
\hline 114 & 0.2844 & 0.011 & 0.3917 & 10.5 \\
\hline 115 & 2.3913 & 0.011 & 0.1483 & 14.5 \\
\hline 116 & 1.0857 & 0.011 & 0.1677 & 12.5 \\
\hline 117 & 4.9631 & 0.011 & 0.7112 & 20.1 \\
\hline 118 & 0.5891 & 0.013 & 0.5727 & 11.8 \\
\hline 119 & 1.8169 & 0.011 & 0.4276 & 13.5 \\
\hline 120 & 1.3692 & 0.011 & 0.2257 & 12.6 \\
\hline 121 & 0.0432 & 0.014 & 0.1173 & 9.8 \\
\hline 122 & 1.4164 & 0.011 & 0.6597 & 12.3 \\
\hline 123 & 1.8143 & 0.012 & 0.6238 & 13.6 \\
\hline 124 & 0.0846 & 0.010 & 0.7156 & 9.7 \\
\hline 125 & 0.0069 & 0.012 & 0.4976 & 9.6 \\
\hline 126 & 2.9924 & 0.010 & 0.7486 & 14.9 \\
\hline 127 & 3.6239 & 0.012 & 0.3657 & 16.7 \\
\hline 128 & 3.0247 & 0.012 & 0.2936 & 15.0 \\
\hline 129 & 3.9680 & 0.011 & 0.6050 & 17.3 \\
\hline 130 & 4.3590 & 0.011 & 0.5049 & 18.0 \\
\hline 131 & 0.0565 & 0.013 & 0.4973 & 9.4 \\
\hline 132 & 2.4153 & 0.011 & 0.3813 & 13.8 \\
\hline 133 & 0.0533 & 0.011 & 0.3310 & 9.6 \\
\hline 134 & 0.5943 & 0.011 & 0.9709 & 10.9 \\
\hline 135 & 0.5597 & 0.011 & 0.2706 & 10.4 \\
\hline 136 & 2.6142 & 0.010 & 0.3255 & 14.1 \\
\hline 137 & 1.8084 & 0.010 & 0.6916 & 12.5 \\
\hline 138 & 4.7018 & 0.010 & 0.8318 & 17.8 \\
\hline 139 & 0.3418 & 0.008 & 0.7422 & 9.5 \\
\hline 140 & 0.0026 & 0.011 & 0.8688 & 9.1 \\
\hline 141 & 1.2124 & 0.012 & 0.7828 & 12.9 \\
\hline 142 & 4.8318 & 0.011 & 0.7306 & 18.1 \\
\hline 143 & 0.5695 & 0.008 & 0.4827 & 9.9 \\
\hline 144 & 0.6245 & 0.010 & 0.5124 & 10.1 \\
\hline 145 & 2.5468 & 0.009 & 0.2065 & 13.1 \\
\hline 146 & 0.1052 & 0.010 & 0.6602 & 9.0 \\
\hline 147 & 0.0336 & 0.010 & 0.5657 & 8.4 \\
\hline 148 & 0.1134 & 0.008 & 0.2306 & 9.0 \\
\hline 149 & 1.5992 & 0.009 & 0.8162 & 11.7 \\
\hline 150 & 0.0214 & 0.010 & 0.0713 & 8.6 \\
\hline 151 & 0.0449 & 0.009 & 0.0795 & 9.2 \\
\hline 152 & 0.5877 & 0.011 & 0.1086 & 10.1 \\
\hline 153 & 0.5797 & 0.009 & 0.1969 & 10.0 \\
\hline 154 & 1.8645 & 0.009 & 0.9255 & 11.9 \\
\hline 155 & 1.2036 & 0.012 & 0.7640 & 12.9 \\
\hline 156 & 1.3178 & 0.009 & 0.8759 & 11.2 \\
\hline 157 & 0.5740 & 0.009 & 0.2844 & 9.9 \\
\hline 158 & 0.0288 & 0.007 & 0.6727 & 8.8 \\
\hline 159 & 4.2278 & 0.010 & 0.6653 & 16.5 \\
\hline 160 & 4.8369 & 0.009 & 0.8982 & 17.0 \\
\hline 161 & 1.7958 & 0.009 & 0.3709 & 11.6 \\
\hline
\end{tabular}


Table A1. Continued.

\begin{tabular}{|c|c|c|c|c|}
\hline$N$ & $F$ & $A$ & $P$ & SNR \\
\hline 162 & 1.0052 & 0.009 & 0.6319 & 10. \\
\hline 163 & 0.9951 & 0.008 & 0.3070 & 10. \\
\hline 164 & 0.0895 & 0.012 & 0.7737 & 8. \\
\hline 165 & 0.0143 & 0.007 & 0.5967 & 8. \\
\hline 166 & 3.4937 & 0.009 & 0.4420 & 14. \\
\hline 167 & 3.6173 & 0.008 & 0.7603 & 14. \\
\hline 168 & 0.1378 & 0.010 & 0.3321 & 8. \\
\hline 169 & 0.0480 & 0.007 & 0.8814 & 8. \\
\hline 170 & 0.0186 & 0.006 & 0.1057 & 8. \\
\hline 171 & 0.0509 & 0.008 & 0.9190 & 8. \\
\hline 172 & 0.0803 & 0.010 & 0.6598 & 8. \\
\hline 173 & 0.0087 & 0.013 & 0.7656 & 8. \\
\hline 174 & 2.5259 & 0.008 & 0.5191 & 12. \\
\hline 175 & 2.3384 & 0.008 & 0.8083 & 11. \\
\hline 176 & 1.9985 & 0.009 & 0.7772 & 11. \\
\hline 177 & 4.4100 & 0.009 & 0.5855 & 15. \\
\hline 178 & 1.2272 & 0.009 & 0.9901 & 10. \\
\hline 179 & 0.5964 & 0.008 & 0.1089 & 9. \\
\hline 180 & 0.6187 & 0.012 & 0.3725 & 10. \\
\hline 181 & 0.6206 & 0.011 & 0.0193 & 10. \\
\hline 182 & 0.0315 & 0.014 & 0.7970 & 8. \\
\hline 183 & 0.0623 & 0.006 & 0.0421 & 8. \\
\hline 184 & 1.2171 & 0.010 & 0.5839 & 10. \\
\hline 185 & 1.1873 & 0.007 & 0.8383 & 10. \\
\hline 186 & 1.2160 & 0.009 & 0.6245 & 10. \\
\hline 187 & 0.0263 & 0.012 & 0.1299 & 8. \\
\hline 188 & 4.2328 & 0.010 & 0.9689 & 14. \\
\hline 189 & 4.9498 & 0.007 & 0.6767 & 15. \\
\hline 190 & 0.6314 & 0.004 & 0.0839 & 8.8 \\
\hline 191 & 1.9218 & 0.008 & 0.8825 & 10. \\
\hline 192 & 3.3306 & 0.008 & 0.2377 & 13. \\
\hline 193 & 3.6288 & 0.007 & 0.7552 & 13. \\
\hline 194 & 4.0977 & 0.008 & 0.3866 & 14. \\
\hline 195 & 0.2130 & 0.008 & 0.6613 & 8. \\
\hline 196 & 0.0049 & 0.010 & 0.9493 & 8. \\
\hline 197 & 0.0986 & 0.006 & 0.4770 & 7. \\
\hline 198 & 2.4135 & 0.006 & 0.6587 & 11. \\
\hline 199 & 4.8254 & 0.008 & 0.1253 & 15. \\
\hline 200 & 3.6223 & 0.007 & 0.8083 & 13. \\
\hline 201 & 1.2242 & 0.007 & 0.4654 & 9. \\
\hline 202 & 0.0368 & 0.007 & 0.6030 & 7. \\
\hline 203 & 0.5729 & 0.007 & 0.7046 & 8. \\
\hline 204 & 0.6579 & 0.007 & 0.6780 & 8. \\
\hline 205 & 0.1018 & 0.006 & 0.3971 & 7. \\
\hline 206 & 0.6838 & 0.008 & 0.5788 & 8. \\
\hline 207 & 0.1426 & 0.004 & 0.4217 & 7. \\
\hline 208 & 0.6423 & 0.010 & 0.2468 & 8. \\
\hline 209 & 1.8185 & 0.008 & 0.1807 & 9. \\
\hline 210 & 0.0864 & 0.010 & 0.2549 & 7. \\
\hline 211 & 0.3955 & 0.007 & 0.6943 & 7. \\
\hline 212 & 0.0670 & 0.007 & 0.2321 & 7. \\
\hline 213 & 3.0194 & 0.008 & 0.8203 & 11. \\
\hline 214 & 2.2032 & 0.007 & 0.1224 & 10. \\
\hline 215 & 0.0063 & 0.011 & 0.2367 & 7. \\
\hline 216 & 0.0630 & 0.008 & 0.2310 & 7. \\
\hline 217 & 0.0156 & 0.010 & 0.9619 & 7. \\
\hline 218 & 0.0198 & 0.009 & 0.9606 & 8. \\
\hline 219 & 0.6261 & 0.006 & 0.1145 & 8. \\
\hline 220 & 0.5823 & 0.010 & 0.3587 & 8. \\
\hline 221 & 2.4091 & 0.008 & 0.4653 & 10. \\
\hline 222 & 1.8695 & 0.007 & 0.6241 & 9. \\
\hline 223 & 2.4186 & 0.008 & 0.1687 & 10. \\
\hline
\end{tabular}

Table A1. Continued.

\begin{tabular}{|c|c|c|c|c|}
\hline$N$ & $F$ & $A$ & $P$ & SNR \\
\hline 224 & 0.6488 & 0.007 & 0.7964 & 8.0 \\
\hline 225 & 0.0397 & 0.007 & 0.1003 & 7.3 \\
\hline 226 & 0.0354 & 0.006 & 0.4586 & 7.6 \\
\hline 227 & 0.0938 & 0.007 & 0.1194 & 7.1 \\
\hline 228 & 1.1982 & 0.007 & 0.5649 & 8.6 \\
\hline 229 & 0.9672 & 0.006 & 0.0567 & 8.3 \\
\hline 230 & 2.8896 & 0.007 & 0.9090 & 10.8 \\
\hline 231 & 1.2020 & 0.009 & 0.0116 & 9.4 \\
\hline 232 & 3.1509 & 0.007 & 0.9775 & 11.2 \\
\hline 233 & 0.5869 & 0.009 & 0.8703 & 8.1 \\
\hline 234 & 1.2355 & 0.007 & 0.5330 & 8.6 \\
\hline 235 & 3.0208 & 0.007 & 0.4495 & 11.1 \\
\hline 236 & 2.7926 & 0.006 & 0.8003 & 10.5 \\
\hline 237 & 0.3717 & 0.007 & 0.5017 & 7.2 \\
\hline 238 & 1.2140 & 0.008 & 0.0111 & 8.7 \\
\hline 239 & 0.0222 & 0.009 & 0.8167 & 6.8 \\
\hline 240 & 0.5759 & 0.007 & 0.5043 & 7.7 \\
\hline 241 & 0.5772 & 0.006 & 0.1883 & 8.2 \\
\hline 242 & 0.0739 & 0.006 & 0.3209 & 6.7 \\
\hline 243 & 0.0574 & 0.005 & 0.2890 & 7.2 \\
\hline 244 & 0.0077 & 0.006 & 0.9595 & 7.0 \\
\hline 245 & 4.4674 & 0.006 & 0.2380 & 12.8 \\
\hline 246 & 0.9993 & 0.006 & 0.4145 & 8.1 \\
\hline 247 & 0.0322 & 0.007 & 0.6979 & 6.5 \\
\hline 248 & 1.1968 & 0.006 & 0.3491 & 8.2 \\
\hline 249 & 0.6349 & 0.004 & 0.1773 & 7.5 \\
\hline 250 & 0.0787 & 0.006 & 0.2070 & 6.8 \\
\hline 251 & 4.6394 & 0.006 & 0.1738 & 12.8 \\
\hline 252 & 2.0074 & 0.006 & 0.1696 & 9.1 \\
\hline 253 & 0.6599 & 0.006 & 0.8010 & 7.3 \\
\hline 254 & 1.2186 & 0.006 & 0.7455 & 8.1 \\
\hline 255 & 2.4178 & 0.007 & 0.2341 & 10.0 \\
\hline 256 & 0.1532 & 0.006 & 0.5155 & 6.4 \\
\hline 257 & 3.6261 & 0.008 & 0.0635 & 11.5 \\
\hline 258 & 1.8128 & 0.006 & 0.4256 & 9.2 \\
\hline 259 & 0.0178 & 0.009 & 0.9468 & 7.1 \\
\hline 260 & 0.1111 & 0.007 & 0.2124 & 6.6 \\
\hline 261 & 0.0609 & 0.009 & 0.2394 & 6.6 \\
\hline 262 & 0.1258 & 0.009 & 0.3570 & 6.6 \\
\hline 263 & 1.2226 & 0.008 & 0.0595 & 8.1 \\
\hline 264 & 0.6411 & 0.006 & 0.8622 & 7.2 \\
\hline 265 & 0.6323 & 0.008 & 0.4660 & 7.4 \\
\hline 266 & 3.0235 & 0.007 & 0.6443 & 10.0 \\
\hline 267 & 4.8395 & 0.006 & 0.7207 & 12.3 \\
\hline 268 & 3.0726 & 0.005 & 0.2607 & 9.9 \\
\hline 269 & 0.0494 & 0.007 & 0.2219 & 6.7 \\
\hline 270 & 0.0755 & 0.009 & 0.6666 & 6.8 \\
\hline 271 & 0.5363 & 0.004 & 0.8838 & 6.9 \\
\hline 272 & 1.0775 & 0.006 & 0.5053 & 7.6 \\
\hline 273 & 4.2214 & 0.006 & 0.7295 & 11.4 \\
\hline 274 & 1.9485 & 0.006 & 0.3600 & 8.5 \\
\hline 275 & 2.0246 & 0.007 & 0.7458 & 8.6 \\
\hline 276 & 0.5568 & 0.007 & 0.1948 & 6.7 \\
\hline 277 & 0.5676 & 0.005 & 0.3004 & 6.8 \\
\hline 278 & 0.4949 & 0.006 & 0.3021 & 6.7 \\
\hline 279 & 4.8282 & 0.006 & 0.0358 & 11.8 \\
\hline 280 & 4.8343 & 0.007 & 0.7484 & 12.1 \\
\hline 281 & 0.1558 & 0.006 & 0.2316 & 6.2 \\
\hline 282 & 0.1548 & 0.007 & 0.8164 & 7.1 \\
\hline 283 & 0.1661 & 0.006 & 0.3987 & 6.2 \\
\hline 284 & 0.5587 & 0.006 & 0.9196 & 7.1 \\
\hline 285 & 0.0972 & 0.005 & 0.5152 & 6.0 \\
\hline
\end{tabular}


Table A1. Continued.

\begin{tabular}{|c|c|c|c|c|}
\hline$N$ & $F$ & $A$ & $P$ & SNR \\
\hline 286 & 0.0644 & 0.005 & 0.9109 & 6.2 \\
\hline 287 & 1.9416 & 0.006 & 0.6954 & 8.5 \\
\hline 288 & 1.1844 & 0.006 & 0.0778 & 7.4 \\
\hline 289 & 1.2148 & 0.007 & 0.6590 & 7.7 \\
\hline 290 & 2.4615 & 0.005 & 0.7649 & 8.7 \\
\hline 291 & 1.1953 & 0.005 & 0.1796 & 7.5 \\
\hline 292 & 0.1286 & 0.006 & 0.4343 & 6.2 \\
\hline 293 & 2.4120 & 0.006 & 0.6780 & 8.6 \\
\hline 294 & 2.4219 & 0.006 & 0.3595 & 9.1 \\
\hline 295 & 0.6379 & 0.004 & 0.7334 & 6.8 \\
\hline 296 & 4.2270 & 0.006 & 0.2032 & 10.9 \\
\hline 297 & 0.1249 & 0.005 & 0.5003 & 6.2 \\
\hline 298 & 4.8304 & 0.005 & 0.5935 & 11.5 \\
\hline 299 & 3.6203 & 0.006 & 0.6814 & 10.0 \\
\hline 300 & 1.3331 & 0.006 & 0.5969 & 7.6 \\
\hline 301 & 0.1817 & 0.005 & 0.4327 & 6.0 \\
\hline 302 & 2.0261 & 0.005 & 0.5292 & 8.5 \\
\hline 303 & 3.6231 & 0.005 & 0.0308 & 10.3 \\
\hline 304 & 0.6272 & 0.004 & 0.4733 & 6.9 \\
\hline 305 & 0.5521 & 0.006 & 0.5181 & 6.4 \\
\hline 306 & 0.3340 & 0.004 & 0.7556 & 6.1 \\
\hline 307 & 1.8001 & 0.005 & 0.0146 & 7.8 \\
\hline 308 & 4.8848 & 0.005 & 0.8176 & 11.1 \\
\hline 309 & 3.6273 & 0.006 & 0.9809 & 10.2 \\
\hline 310 & 0.6629 & 0.006 & 0.2632 & 6.5 \\
\hline 311 & 0.6367 & 0.004 & 0.9079 & 7.0 \\
\hline 312 & 0.5779 & 0.004 & 0.0337 & 6.7 \\
\hline 313 & 0.5818 & 0.006 & 0.3530 & 7.0 \\
\hline 314 & 2.5455 & 0.005 & 0.4428 & 8.3 \\
\hline 315 & 2.6639 & 0.005 & 0.3763 & 8.5 \\
\hline 316 & 0.5716 & 0.005 & 0.1861 & 6.5 \\
\hline 317 & 0.1264 & 0.005 & 0.8625 & 5.8 \\
\hline 318 & 0.1182 & 0.005 & 0.3956 & 5.9 \\
\hline 319 & 3.6767 & 0.005 & 0.8874 & 9.7 \\
\hline 320 & 1.3373 & 0.005 & 0.4933 & 7.1 \\
\hline 321 & 0.6556 & 0.004 & 0.4132 & 6.5 \\
\hline 322 & 2.2856 & 0.005 & 0.4427 & 8.1 \\
\hline 323 & 1.4763 & 0.005 & 0.9978 & 7.2 \\
\hline 324 & 1.1644 & 0.005 & 0.8101 & 7.0 \\
\hline 325 & 1.1924 & 0.004 & 0.0803 & 7.2 \\
\hline 326 & 1.2013 & 0.005 & 0.5915 & 7.5 \\
\hline 327 & 0.2462 & 0.005 & 0.7426 & 5.9 \\
\hline 328 & 0.1468 & 0.005 & 0.8532 & 5.9 \\
\hline 329 & 0.1618 & 0.005 & 0.2239 & 5.8 \\
\hline 330 & 0.0583 & 0.007 & 0.2740 & 6.2 \\
\hline 331 & 0.5860 & 0.004 & 0.5058 & 7.0 \\
\hline 332 & 0.6462 & 0.006 & 0.1790 & 6.4 \\
\hline 333 & 1.5799 & 0.005 & 0.3919 & 7.3 \\
\hline 334 & 2.4147 & 0.005 & 0.8363 & 8.4 \\
\hline 335 & 3.0223 & 0.005 & 0.4275 & 9.0 \\
\hline 336 & 3.6313 & 0.006 & 0.2562 & 9.5 \\
\hline 337 & 0.1632 & 0.004 & 0.8586 & 5.8 \\
\hline 338 & 3.7341 & 0.005 & 0.4397 & 9.5 \\
\hline 339 & 1.8209 & 0.005 & 0.2187 & 7.6 \\
\hline 340 & 1.8070 & 0.005 & 0.6261 & 7.7 \\
\hline 341 & 0.1010 & 0.007 & 0.7478 & 5.7 \\
\hline 342 & 0.0763 & 0.006 & 0.2092 & 5.7 \\
\hline 343 & 0.0330 & 0.002 & 0.8370 & 6.3 \\
\hline 344 & 0.0377 & 0.007 & 0.2750 & 6.2 \\
\hline 345 & 0.1044 & 0.005 & 0.3752 & 5.8 \\
\hline 346 & 0.6069 & 0.006 & 0.4180 & 6.9 \\
\hline 347 & 0.6444 & 0.008 & 0.2527 & 6.5 \\
\hline
\end{tabular}

Table A1. Continued.

\begin{tabular}{|c|c|c|c|c|}
\hline$N$ & $F$ & A & $P$ & SNR \\
\hline 348 & 0.0032 & 0.007 & 0.6035 & 6.7 \\
\hline 349 & 0.0710 & 0.007 & 0.0744 & 6.0 \\
\hline 350 & 1.2278 & 0.005 & 0.1573 & 7.3 \\
\hline 351 & 1.5472 & 0.005 & 0.6609 & 7.2 \\
\hline 352 & 4.2246 & 0.005 & 0.6882 & 10.2 \\
\hline 353 & 4.9421 & 0.005 & 0.9629 & 10.7 \\
\hline 354 & 4.2356 & 0.005 & 0.0066 & 10.0 \\
\hline 355 & 1.6815 & 0.005 & 0.2665 & 7.3 \\
\hline 356 & 0.0746 & 0.005 & 0.9862 & 6.2 \\
\hline 357 & 0.0954 & 0.006 & 0.5252 & 6.0 \\
\hline 358 & 0.1461 & 0.004 & 0.0825 & 5.9 \\
\hline 359 & 0.1349 & 0.006 & 0.1508 & 5.6 \\
\hline 360 & 0.1538 & 0.005 & 0.2414 & 6.8 \\
\hline 361 & 0.1685 & 0.003 & 0.3625 & 5.9 \\
\hline 362 & 3.0186 & 0.005 & 0.4121 & 8.6 \\
\hline 363 & 3.0253 & 0.005 & 0.3291 & 9.0 \\
\hline 364 & 3.0164 & 0.005 & 0.1152 & 8.6 \\
\hline 365 & 2.4682 & 0.006 & 0.6312 & 9.4 \\
\hline 366 & 2.6966 & 0.005 & 0.9016 & 8.0 \\
\hline 367 & 0.6939 & 0.006 & 0.4330 & 6.2 \\
\hline 368 & 0.7096 & 0.005 & 0.8726 & 6.4 \\
\hline 369 & 0.6475 & 0.005 & 0.5853 & 6.5 \\
\hline 370 & 0.4486 & 0.004 & 0.3846 & 6.0 \\
\hline 371 & 0.1957 & 0.005 & 0.3543 & 5.6 \\
\hline 372 & 0.0682 & 0.007 & 0.6588 & 5.4 \\
\hline 373 & 0.4859 & 0.005 & 0.9815 & 6.0 \\
\hline 374 & 0.5481 & 0.004 & 0.8305 & 6.0 \\
\hline 375 & 0.4665 & 0.004 & 0.0094 & 5.9 \\
\hline 376 & 4.2807 & 0.004 & 0.0670 & 9.6 \\
\hline 377 & 1.3744 & 0.004 & 0.5523 & 6.7 \\
\hline 378 & 2.3970 & 0.004 & 0.0194 & 7.6 \\
\hline 379 & 0.5398 & 0.005 & 0.0810 & 5.9 \\
\hline 380 & 0.6700 & 0.004 & 0.3677 & 6.0 \\
\hline 381 & 0.7127 & 0.004 & 0.9816 & 6.2 \\
\hline 382 & 0.6172 & 0.007 & 0.1135 & 7.0 \\
\hline 383 & 0.6520 & 0.005 & 0.4788 & 6.0 \\
\hline 384 & 3.2654 & 0.004 & 0.9935 & 8.2 \\
\hline 385 & 1.2430 & 0.004 & 0.7093 & 6.4 \\
\hline 386 & 0.0962 & 0.008 & 0.7486 & 5.9 \\
\hline 387 & 0.1095 & 0.004 & 0.6922 & 5.2 \\
\hline 388 & 2.4161 & 0.004 & 0.9073 & 7.4 \\
\hline 389 & 0.4732 & 0.003 & 0.0929 & 5.6 \\
\hline 390 & 0.6307 & 0.006 & 0.1380 & 6.0 \\
\hline 391 & 0.6096 & 0.008 & 0.1899 & 9.0 \\
\hline 392 & 0.6119 & 0.007 & 0.5343 & 7.2 \\
\hline 393 & 0.0780 & 0.004 & 0.7264 & 5.4 \\
\hline 394 & 1.0790 & 0.004 & 0.6642 & 6.2 \\
\hline 395 & 3.4950 & 0.004 & 0.1871 & 8.2 \\
\hline 396 & 1.4275 & 0.004 & 0.7428 & 6.4 \\
\hline 397 & 1.1896 & 0.005 & 0.6146 & 6.3 \\
\hline 398 & 1.2056 & 0.007 & 0.1518 & 8.3 \\
\hline 399 & 0.0242 & 0.006 & 0.5848 & 5.7 \\
\hline 400 & 0.0770 & 0.004 & 0.6703 & 5.9 \\
\hline 401 & 0.0135 & 0.004 & 0.5155 & 5.7 \\
\hline 402 & 0.0463 & 0.005 & 0.7925 & 6.2 \\
\hline 403 & 2.5190 & 0.004 & 0.9394 & 7.3 \\
\hline 404 & 4.7032 & 0.004 & 0.3849 & 9.5 \\
\hline 405 & 4.3381 & 0.004 & 0.6858 & 9.1 \\
\hline 406 & 1.8194 & 0.004 & 0.3103 & 7.1 \\
\hline 407 & 0.5656 & 0.006 & 0.0322 & 5.8 \\
\hline 408 & 2.7636 & 0.004 & 0.0579 & 7.5 \\
\hline 409 & 0.6157 & 0.006 & 0.1911 & 6.9 \\
\hline
\end{tabular}


Table A1. Continued.

\begin{tabular}{|c|c|c|c|c|}
\hline$N$ & $F$ & $A$ & $P$ & SNR \\
\hline 410 & 0.5847 & 0.006 & 0.4052 & 6 \\
\hline 411 & 0.2017 & 0.005 & 0.3462 & 5 \\
\hline 412 & 3.1300 & 0.004 & 0.3363 & 7 \\
\hline 413 & 3.6218 & 0.004 & 0.9463 & 9 \\
\hline 414 & 0.1365 & 0.005 & 0.1730 & \\
\hline 415 & 0.0390 & 0.007 & 0.8282 & 5 \\
\hline 416 & 0.0361 & 0.004 & 0.4996 & 6 \\
\hline 417 & 0.0617 & 0.005 & 0.8663 & 5 \\
\hline 418 & 0.1883 & 0.004 & 0.8337 & 5 \\
\hline 419 & 1.2639 & 0.004 & 0.5090 & 6 \\
\hline 420 & 0.6332 & 0.005 & 0.4889 & 6 \\
\hline 421 & 0.6255 & 0.006 & 0.7199 & 7 \\
\hline 422 & 0.5789 & 0.005 & 0.9912 & 6 \\
\hline 423 & 0.7763 & 0.004 & 0.4223 & 5 \\
\hline 424 & 2.7876 & 0.004 & 0.8879 & 7 \\
\hline 425 & 4.2334 & 0.005 & 0.4100 & 9. \\
\hline 426 & 0.1212 & 0.005 & 0.7227 & 5. \\
\hline 427 & 0.1320 & 0.003 & 0.9835 & 5 \\
\hline 428 & 0.6236 & 0.006 & 0.3066 & 6 \\
\hline 429 & 0.1128 & 0.005 & 0.7081 & 5 \\
\hline 430 & 3.0132 & 0.004 & 0.7375 & 7 \\
\hline 431 & 2.8910 & 0.004 & 0.2992 & 7. \\
\hline 432 & 3.8303 & 0.004 & 0.1565 & 8. \\
\hline 433 & 0.6917 & 0.005 & 0.3599 & 5. \\
\hline 434 & 0.5977 & 0.005 & 0.6593 & 6 \\
\hline 435 & 0.0149 & 0.004 & 0.3717 & 5 \\
\hline 436 & 4.8290 & 0.004 & 0.8955 & 9 \\
\hline 437 & 0.5458 & 0.003 & 0.7050 & 5. \\
\hline 438 & 0.5373 & 0.006 & 0.0530 & 5. \\
\hline 439 & 1.2251 & 0.004 & 0.0145 & 6. \\
\hline 440 & 0.8858 & 0.004 & 0.5837 & 5. \\
\hline 441 & 0.6359 & 0.006 & 0.8781 & 6 \\
\hline 442 & 2.5803 & 0.004 & 0.7516 & 7 \\
\hline 443 & 1.2408 & 0.005 & 0.6651 & 6 \\
\hline 444 & 2.8122 & 0.004 & 0.3518 & 7 \\
\hline 445 & 4.8352 & 0.005 & 0.3182 & 9 \\
\hline 446 & 1.8091 & 0.004 & 0.9382 & 6 \\
\hline 447 & 0.5834 & 0.007 & 0.3102 & 6. \\
\hline 448 & 0.5275 & 0.006 & 0.9830 & 5 \\
\hline 449 & 0.1141 & 0.005 & 0.0340 & 5 \\
\hline 450 & 0.0929 & 0.007 & 0.1916 & 5 \\
\hline 451 & 0.2924 & 0.005 & 0.9228 & 5 \\
\hline 452 & 4.2316 & 0.005 & 0.9844 & 8. \\
\hline 453 & 4.2304 & 0.004 & 0.4969 & 9 \\
\hline 454 & 4.3576 & 0.004 & 0.8228 & 8. \\
\hline 455 & 1.8041 & 0.004 & 0.9325 & 6. \\
\hline 456 & 2.2187 & 0.004 & 0.9185 & 6. \\
\hline 457 & 4.8310 & 0.004 & 0.1935 & 9 \\
\hline 458 & 1.2346 & 0.004 & 0.5441 & 6. \\
\hline 459 & 1.8602 & 0.004 & 0.0884 & 6 \\
\hline 460 & 3.5830 & 0.004 & 0.8596 & 7 \\
\hline 461 & 0.6746 & 0.004 & 0.0340 & 5. \\
\hline 462 & 0.5685 & 0.005 & 0.8404 & 6 \\
\hline 463 & 0.5419 & 0.004 & 0.7589 & 5 \\
\hline 464 & 0.2362 & 0.004 & 0.7916 & 5. \\
\hline 465 & 0.1931 & 0.003 & 0.8427 & 5 \\
\hline 466 & 3.5256 & 0.004 & 0.5599 & 7 \\
\hline 467 & 1.1938 & 0.004 & 0.1118 & 5 \\
\hline 468 & 0.0705 & 0.007 & 0.8385 & 5 \\
\hline 469 & 0.6673 & 0.006 & 0.7820 & 5 \\
\hline 470 & 0.6685 & 0.005 & 0.2409 & 5. \\
\hline 471 & 0.4626 & 0.004 & 0.8015 & 5 \\
\hline
\end{tabular}

Table A1. Continued.

\begin{tabular}{|c|c|c|c|c|}
\hline$N$ & $F$ & $A$ & $P$ & SNR \\
\hline 472 & 4.8361 & 0.004 & 0.9403 & 9.3 \\
\hline 473 & 4.9617 & 0.004 & 0.5742 & 8.9 \\
\hline 474 & 0.0945 & 0.006 & 0.3966 & 5.3 \\
\hline 475 & 1.9475 & 0.004 & 0.4560 & 6.5 \\
\hline 476 & 0.6667 & 0.006 & 0.1897 & 6.1 \\
\hline 477 & 0.6985 & 0.004 & 0.1118 & 5.4 \\
\hline 478 & 0.6803 & 0.004 & 0.6363 & 5.4 \\
\hline 479 & 0.0303 & 0.006 & 0.8319 & 5.3 \\
\hline 480 & 0.7038 & 0.004 & 0.1086 & 5.3 \\
\hline 481 & 0.3299 & 0.004 & 0.7127 & 4.9 \\
\hline 482 & 0.3033 & 0.004 & 0.6330 & 5.0 \\
\hline 483 & 0.1611 & 0.004 & 0.0336 & 4.9 \\
\hline 484 & 0.2035 & 0.004 & 0.1517 & 4.8 \\
\hline 485 & 0.2694 & 0.004 & 0.9749 & 4.8 \\
\hline 486 & 3.9009 & 0.004 & 0.1089 & 7.7 \\
\hline 487 & 0.1649 & 0.004 & 0.5565 & 4.9 \\
\hline 488 & 1.1698 & 0.003 & 0.7949 & 5.4 \\
\hline 489 & 1.2301 & 0.004 & 0.3618 & 5.6 \\
\hline 490 & 1.5136 & 0.003 & 0.8284 & 5.6 \\
\hline 491 & 0.5192 & 0.004 & 0.6906 & 4.9 \\
\hline 492 & 0.5221 & 0.003 & 0.0884 & 5.0 \\
\hline 493 & 0.3680 & 0.003 & 0.2712 & 4.8 \\
\hline 494 & 0.4213 & 0.003 & 0.8097 & 5.0 \\
\hline 495 & 0.1854 & 0.004 & 0.4820 & 4.7 \\
\hline 496 & 0.4602 & 0.003 & 0.5508 & 5.0 \\
\hline 497 & 0.2642 & 0.004 & 0.2734 & 4.6 \\
\hline 498 & 0.0652 & 0.008 & 0.0828 & 5.4 \\
\hline 499 & 0.0732 & 0.004 & 0.9467 & 4.8 \\
\hline 500 & 0.0555 & 0.007 & 0.3539 & 5.0 \\
\hline 501 & 0.1031 & 0.004 & 0.4012 & 5.0 \\
\hline 502 & 0.1074 & 0.005 & 0.6009 & 4.7 \\
\hline 503 & 4.0991 & 0.003 & 0.6757 & 7.6 \\
\hline 504 & 0.5535 & 0.003 & 0.4260 & 5.0 \\
\hline 505 & 2.8701 & 0.003 & 0.3971 & 6.6 \\
\hline 506 & 1.9436 & 0.004 & 0.2756 & 6.1 \\
\hline 507 & 4.8374 & 0.004 & 0.7261 & 9.0 \\
\hline 508 & 4.0504 & 0.003 & 0.7416 & 7.6 \\
\hline 509 & 3.0118 & 0.003 & 0.3390 & 6.7 \\
\hline 510 & 0.5441 & 0.003 & 0.0740 & 5.0 \\
\hline 511 & 0.4287 & 0.004 & 0.6468 & 4.8 \\
\hline 512 & 0.7063 & 0.003 & 0.3598 & 5.0 \\
\hline 513 & 2.4231 & 0.004 & 0.6310 & 6.4 \\
\hline 514 & 1.2313 & 0.004 & 0.4308 & 5.5 \\
\hline 515 & 1.8756 & 0.003 & 0.3359 & 5.8 \\
\hline 516 & 1.1806 & 0.004 & 0.1709 & 5.4 \\
\hline 517 & 0.1280 & 0.005 & 0.5063 & 4.8 \\
\hline 518 & 0.1297 & 0.005 & 0.3037 & 5.5 \\
\hline 519 & 0.1899 & 0.004 & 0.9423 & 4.8 \\
\hline 520 & 0.0457 & 0.005 & 0.1781 & 5.0 \\
\hline 521 & 2.0281 & 0.003 & 0.1735 & 5.9 \\
\hline 522 & 0.1244 & 0.005 & 0.9761 & 4.8 \\
\hline 523 & 0.1475 & 0.004 & 0.1391 & 4.5 \\
\hline 524 & 0.1595 & 0.004 & 0.5191 & 4.8 \\
\hline 525 & 0.5283 & 0.004 & 0.5056 & 5.0 \\
\hline 526 & 0.5327 & 0.004 & 0.6999 & 4.9 \\
\hline 527 & 1.2648 & 0.004 & 0.9720 & 5.6 \\
\hline 528 & 1.2326 & 0.004 & 0.1543 & 5.5 \\
\hline 529 & 1.2165 & 0.006 & 0.9131 & 7.4 \\
\hline 530 & 1.2177 & 0.004 & 0.0758 & 6.4 \\
\hline 531 & 3.6302 & 0.003 & 0.3329 & 7.3 \\
\hline 532 & 1.0497 & 0.003 & 0.6360 & 5.1 \\
\hline 533 & 1.2232 & 0.004 & 0.6588 & 6.2 \\
\hline
\end{tabular}


Table A1. Continued.

\begin{tabular}{|c|c|c|c|c|}
\hline$N$ & $F$ & $A$ & $P$ & SNR \\
\hline 534 & 1.2741 & 0.003 & 0.7266 & 5.4 \\
\hline 535 & 2.7576 & 0.003 & 0.1622 & 6.2 \\
\hline 536 & 0.1188 & 0.002 & 0.7721 & 4.8 \\
\hline 537 & 1.6829 & 0.003 & 0.8629 & 5.6 \\
\hline 538 & 4.1255 & 0.003 & 0.6723 & 7.3 \\
\hline 539 & 2.0233 & 0.004 & 0.9011 & 5.9 \\
\hline 540 & 2.0022 & 0.003 & 0.7112 & 5.8 \\
\hline 541 & 0.5383 & 0.004 & 0.3036 & 5.1 \\
\hline 542 & 1.2296 & 0.004 & 0.4024 & 6.5 \\
\hline 543 & 0.6535 & 0.005 & 0.5351 & 5.0 \\
\hline 544 & 0.4335 & 0.003 & 0.3702 & 4.7 \\
\hline 545 & 0.4434 & 0.003 & 0.2898 & 4.7 \\
\hline 546 & 0.4587 & 0.004 & 0.4281 & 4.8 \\
\hline 547 & 0.5246 & 0.003 & 0.5612 & 5.0 \\
\hline 548 & 1.1910 & 0.004 & 0.9239 & 5.3 \\
\hline 549 & 0.4798 & 0.004 & 0.5885 & 4.8 \\
\hline 550 & 0.0913 & 0.005 & 0.3688 & 4.6 \\
\hline 551 & 0.0795 & 0.006 & 0.8252 & 5.0 \\
\hline 552 & 0.1801 & 0.003 & 0.0581 & 4.7 \\
\hline 553 & 1.4486 & 0.003 & 0.7212 & 5.3 \\
\hline 554 & 2.3145 & 0.003 & 0.8615 & 5.9 \\
\hline 555 & 0.1409 & 0.004 & 0.2184 & 4.5 \\
\hline 556 & 0.1437 & 0.004 & 0.1211 & 4.8 \\
\hline 557 & 0.1703 & 0.003 & 0.8933 & 4.6 \\
\hline 558 & 0.0870 & 0.006 & 0.5919 & 5.0 \\
\hline 559 & 0.0228 & 0.004 & 0.8220 & 4.8 \\
\hline 560 & 2.5159 & 0.003 & 0.2398 & 5.9 \\
\hline 561 & 1.6358 & 0.003 & 0.9488 & 5.4 \\
\hline 562 & 0.2373 & 0.004 & 0.6719 & 4.7 \\
\hline 563 & 0.3482 & 0.003 & 0.6624 & 4.6 \\
\hline 564 & 2.4675 & 0.003 & 0.4860 & 6.1 \\
\hline 565 & 0.4299 & 0.004 & 0.1855 & 4.6 \\
\hline 566 & 0.1220 & 0.003 & 0.7294 & 4.6 \\
\hline 567 & 0.6679 & 0.005 & 0.4403 & 5.4 \\
\hline 568 & 0.5514 & 0.003 & 0.6977 & 4.8 \\
\hline 569 & 0.1510 & 0.003 & 0.6110 & 4.6 \\
\hline 570 & 0.0880 & 0.004 & 0.0630 & 5.1 \\
\hline 571 & 0.1727 & 0.004 & 0.9597 & 4.6 \\
\hline 572 & 0.0193 & 0.005 & 0.0172 & 5.2 \\
\hline 573 & 1.2469 & 0.003 & 0.1794 & 5.2 \\
\hline 574 & 2.4113 & 0.003 & 0.7440 & 5.9 \\
\hline 575 & 0.6451 & 0.003 & 0.6764 & 5.1 \\
\hline 576 & 0.7395 & 0.004 & 0.9102 & 4.8 \\
\hline 577 & 0.7075 & 0.003 & 0.6798 & 4.9 \\
\hline 578 & 1.8285 & 0.003 & 0.3248 & 5.5 \\
\hline 579 & 0.7002 & 0.003 & 0.8695 & 4.8 \\
\hline 580 & 0.7624 & 0.003 & 0.9665 & 4.8 \\
\hline 581 & 0.6500 & 0.003 & 0.8338 & 5.1 \\
\hline 582 & 0.5005 & 0.003 & 0.4009 & 4.7 \\
\hline 583 & 0.5097 & 0.004 & 0.7355 & 4.7 \\
\hline 584 & 0.1838 & 0.004 & 0.3237 & 4.4 \\
\hline 585 & 4.2229 & 0.002 & 0.0815 & 7.1 \\
\hline 586 & 0.2413 & 0.003 & 0.9250 & 4.3 \\
\hline 587 & 3.2706 & 0.003 & 0.4784 & 6.2 \\
\hline 588 & 0.6871 & 0.003 & 0.5815 & 4.7 \\
\hline 589 & 1.9979 & 0.003 & 0.9963 & 5.6 \\
\hline 590 & 0.2699 & 0.003 & 0.8092 & 4.7 \\
\hline 591 & 0.0172 & 0.005 & 0.0429 & 4.9 \\
\hline 592 & 3.6189 & 0.003 & 0.2065 & 6.4 \\
\hline 593 & 3.6282 & 0.003 & 0.9859 & 7.2 \\
\hline 594 & 3.6195 & 0.003 & 0.5458 & 6.7 \\
\hline 595 & 1.1290 & 0.003 & 0.3133 & 4.9 \\
\hline
\end{tabular}

Table A1. Continued.

\begin{tabular}{|c|c|c|c|c|}
\hline$N$ & $F$ & A & $P$ & SNR \\
\hline 596 & 2.2870 & 0.003 & 0.4770 & 5.6 \\
\hline 597 & 1.1522 & 0.003 & 0.4301 & 4.9 \\
\hline 598 & 4.2264 & 0.003 & 0.4045 & 7.1 \\
\hline 599 & 0.8963 & 0.003 & 0.7366 & 4.7 \\
\hline 600 & 0.4467 & 0.003 & 0.6903 & 4.5 \\
\hline 601 & 0.4499 & 0.003 & 0.9200 & 4.6 \\
\hline 602 & 0.5465 & 0.004 & 0.9503 & 4.8 \\
\hline 603 & 0.5610 & 0.004 & 0.6445 & 4.9 \\
\hline 604 & 0.6393 & 0.004 & 0.5014 & 5.1 \\
\hline 605 & 0.5499 & 0.004 & 0.6060 & 4.8 \\
\hline 606 & 0.5237 & 0.004 & 0.2401 & 5.1 \\
\hline 607 & 0.8415 & 0.003 & 0.1400 & 4.7 \\
\hline 608 & 0.1488 & 0.003 & 0.3449 & 4.6 \\
\hline 609 & 0.0056 & 0.005 & 0.1989 & 5.5 \\
\hline 610 & 0.1081 & 0.004 & 0.6553 & 4.8 \\
\hline 611 & 1.8214 & 0.003 & 0.4326 & 6.3 \\
\hline 612 & 0.0407 & 0.005 & 0.0419 & 4.9 \\
\hline 613 & 0.0276 & 0.004 & 0.0953 & 5.5 \\
\hline 614 & 0.1749 & 0.004 & 0.7462 & 4.4 \\
\hline 615 & 0.1999 & 0.004 & 0.2315 & 4.5 \\
\hline 616 & 3.8855 & 0.003 & 0.1700 & 6.4 \\
\hline 617 & 0.4890 & 0.003 & 0.9331 & 4.6 \\
\hline 618 & 0.5404 & 0.004 & 0.2466 & 5.2 \\
\hline 619 & 0.7427 & 0.003 & 0.1378 & 4.6 \\
\hline 620 & 0.5490 & 0.003 & 0.4207 & 5.1 \\
\hline 621 & 2.5877 & 0.003 & 0.1844 & 5.6 \\
\hline 622 & 2.7741 & 0.003 & 0.7614 & 5.6 \\
\hline 623 & 1.2389 & 0.003 & 0.2676 & 4.9 \\
\hline 624 & 0.7211 & 0.003 & 0.4624 & 4.6 \\
\hline 625 & 0.7292 & 0.003 & 0.0699 & 4.6 \\
\hline 626 & 0.5024 & 0.003 & 0.4594 & 4.5 \\
\hline 627 & 0.7781 & 0.003 & 0.1803 & 4.6 \\
\hline 628 & 0.7586 & 0.003 & 0.1115 & 4.6 \\
\hline 629 & 2.0355 & 0.003 & 0.9931 & 5.3 \\
\hline 630 & 4.6816 & 0.003 & 0.5198 & 6.9 \\
\hline 631 & 0.7543 & 0.003 & 0.4304 & 4.5 \\
\hline 632 & 0.7321 & 0.003 & 0.9189 & 4.6 \\
\hline 633 & 1.8888 & 0.003 & 0.5241 & 5.1 \\
\hline 634 & 3.0178 & 0.003 & 0.8478 & 5.9 \\
\hline 635 & 2.5127 & 0.003 & 0.0020 & 5.5 \\
\hline 636 & 0.3277 & 0.003 & 0.4628 & 4.3 \\
\hline 637 & 1.3621 & 0.003 & 0.6154 & 4.8 \\
\hline 638 & 0.5667 & 0.004 & 0.1746 & 5.0 \\
\hline 639 & 4.8276 & 0.003 & 0.3592 & 7.0 \\
\hline 640 & 1.9965 & 0.003 & 0.7240 & 5.2 \\
\hline 641 & 2.0227 & 0.003 & 0.3445 & 5.4 \\
\hline 642 & 3.0476 & 0.003 & 0.8341 & 5.7 \\
\hline 643 & 1.8024 & 0.003 & 0.1989 & 5.2 \\
\hline 644 & 0.0853 & 0.003 & 0.1046 & 4.4 \\
\hline 645 & 0.4362 & 0.003 & 0.3771 & 4.4 \\
\hline 646 & 0.9193 & 0.003 & 0.7840 & 4.5 \\
\hline 647 & 0.5041 & 0.003 & 0.7596 & 4.6 \\
\hline 648 & 0.3401 & 0.003 & 0.7471 & 4.2 \\
\hline 649 & 0.8521 & 0.003 & 0.9344 & 4.5 \\
\hline 650 & 2.4583 & 0.003 & 0.2607 & 5.3 \\
\hline 651 & 1.2073 & 0.004 & 0.7362 & 6.0 \\
\hline 652 & 1.2888 & 0.003 & 0.1126 & 4.8 \\
\hline 653 & 1.1932 & 0.003 & 0.8973 & 4.9 \\
\hline 654 & 1.2812 & 0.003 & 0.9554 & 4.8 \\
\hline 655 & 1.2681 & 0.003 & 0.2097 & 4.8 \\
\hline 656 & 0.2740 & 0.003 & 0.1674 & 4.2 \\
\hline 657 & 0.2670 & 0.003 & 0.7951 & 4.3 \\
\hline
\end{tabular}


Table A1. Continued.

\begin{tabular}{|c|c|c|c|c|}
\hline$N$ & $F$ & $A$ & $P$ & SNR \\
\hline 658 & 0.3104 & 0.003 & 0.4277 & 4.3 \\
\hline 659 & 0.3823 & 0.003 & 0.4068 & 4.3 \\
\hline 660 & 0.3237 & 0.004 & 0.9759 & 4.3 \\
\hline 661 & 0.3218 & 0.003 & 0.3380 & 4.4 \\
\hline 662 & 0.2936 & 0.003 & 0.3850 & 4.3 \\
\hline 663 & 0.2917 & 0.003 & 0.0415 & 4.4 \\
\hline 664 & 0.6267 & 0.004 & 0.7148 & 6.0 \\
\hline 665 & 0.0980 & 0.003 & 0.8208 & 4.9 \\
\hline 666 & 0.2730 & 0.003 & 0.6144 & 4.6 \\
\hline 667 & 0.5753 & 0.003 & 0.1982 & 4.8 \\
\hline 668 & 2.4105 & 0.003 & 0.8714 & 5.4 \\
\hline 669 & 3.7541 & 0.003 & 0.6437 & 7.1 \\
\hline 670 & 3.2042 & 0.003 & 0.1118 & 5.5 \\
\hline 671 & 3.0146 & 0.003 & 0.5768 & 5.7 \\
\hline 672 & 3.0274 & 0.003 & 0.4952 & 5.7 \\
\hline 673 & 3.0283 & 0.003 & 0.5680 & 6.0 \\
\hline 674 & 4.5341 & 0.003 & 0.3938 & 6.5 \\
\hline 675 & 1.3341 & 0.003 & 0.6482 & 4.9 \\
\hline 676 & 0.3005 & 0.003 & 0.5771 & 4.2 \\
\hline 677 & 1.2216 & 0.003 & 0.0587 & 5.2 \\
\hline 678 & 3.9971 & 0.003 & 0.4223 & 6.1 \\
\hline 679 & 1.8259 & 0.003 & 0.3384 & 5.0 \\
\hline 680 & 1.8076 & 0.003 & 0.8685 & 5.2 \\
\hline 681 & 2.8270 & 0.003 & 0.5419 & 5.4 \\
\hline 682 & 0.4640 & 0.002 & 0.4254 & 4.3 \\
\hline 683 & 0.4567 & 0.003 & 0.5376 & 4.4 \\
\hline 684 & 0.0664 & 0.004 & 0.5410 & 4.8 \\
\hline 685 & 4.8297 & 0.003 & 0.6131 & 6.8 \\
\hline 686 & 0.0549 & 0.004 & 0.5179 & 4.8 \\
\hline 687 & 0.0425 & 0.004 & 0.6134 & 4.5 \\
\hline 688 & 0.3584 & 0.003 & 0.0050 & 4.3 \\
\hline 689 & 0.1907 & 0.003 & 0.9363 & 4.6 \\
\hline 690 & 0.1162 & 0.003 & 0.8436 & 4.8 \\
\hline 691 & 0.3911 & 0.003 & 0.3221 & 4.3 \\
\hline 692 & 0.6542 & 0.003 & 0.7953 & 4.6 \\
\hline 693 & 0.1395 & 0.003 & 0.6188 & 4.3 \\
\hline 694 & 0.2577 & 0.003 & 0.0831 & 4.2 \\
\hline 695 & 0.1088 & 0.003 & 0.7113 & 4.6 \\
\hline 696 & 2.0526 & 0.003 & 0.3691 & 5.1 \\
\hline
\end{tabular}

Table A1. Continued.

\begin{tabular}{|c|c|c|c|c|}
\hline$N$ & $F$ & $A$ & $P$ & SNR \\
\hline 697 & 1.9362 & 0.003 & 0.8072 & 5.0 \\
\hline 698 & 1.3772 & 0.003 & 0.8655 & 4.7 \\
\hline 699 & 2.4126 & 0.003 & 0.1311 & 5.4 \\
\hline 700 & 0.6570 & 0.003 & 0.2109 & 4.9 \\
\hline 701 & 0.5151 & 0.003 & 0.4084 & 4.4 \\
\hline 702 & 0.8439 & 0.003 & 0.7323 & 4.3 \\
\hline 703 & 1.0644 & 0.003 & 0.8552 & 4.5 \\
\hline 704 & 1.8472 & 0.003 & 0.5657 & 4.9 \\
\hline 705 & 4.2238 & 0.003 & 0.9221 & 6.5 \\
\hline 706 & 0.9428 & 0.003 & 0.3426 & 4.3 \\
\hline 707 & 0.6134 & 0.004 & 0.3359 & 5.1 \\
\hline 708 & 0.5909 & 0.003 & 0.1009 & 4.9 \\
\hline 709 & 0.3504 & 0.003 & 0.7099 & 4.0 \\
\hline 710 & 0.3447 & 0.003 & 0.9244 & 4.2 \\
\hline 711 & 0.6714 & 0.004 & 0.3836 & 4.5 \\
\hline 712 & 0.6435 & 0.003 & 0.1370 & 5.3 \\
\hline 713 & 0.5451 & 0.003 & 0.1752 & 4.8 \\
\hline 714 & 0.6725 & 0.003 & 0.7879 & 4.7 \\
\hline 715 & 0.6979 & 0.003 & 0.8107 & 4.7 \\
\hline 716 & 0.7168 & 0.003 & 0.7007 & 4.4 \\
\hline 717 & 0.6623 & 0.003 & 0.1877 & 4.9 \\
\hline 718 & 3.9300 & 0.003 & 0.8226 & 5.9 \\
\hline 719 & 1.2402 & 0.003 & 0.0255 & 4.7 \\
\hline 720 & 1.2586 & 0.003 & 0.6966 & 4.7 \\
\hline 721 & 0.5260 & 0.003 & 0.7856 & 4.5 \\
\hline 722 & 2.8341 & 0.002 & 0.3379 & 5.2 \\
\hline 723 & 0.2627 & 0.003 & 0.1238 & 4.1 \\
\hline 724 & 0.5121 & 0.003 & 0.0645 & 4.4 \\
\hline 725 & 0.7406 & 0.003 & 0.0371 & 4.5 \\
\hline 726 & 0.5621 & 0.003 & 0.6052 & 4.7 \\
\hline 727 & 0.4930 & 0.003 & 0.8863 & 4.3 \\
\hline 728 & 1.8162 & 0.003 & 0.0778 & 5.1 \\
\hline 729 & 0.8539 & 0.002 & 0.1619 & 4.3 \\
\hline 730 & 1.2447 & 0.003 & 0.8448 & 4.7 \\
\hline 731 & 3.9228 & 0.002 & 0.3068 & 5.7 \\
\hline 732 & 1.3589 & 0.002 & 0.2463 & 4.5 \\
\hline 733 & 3.2856 & 0.002 & 0.2462 & 5.3 \\
\hline 734 & 3.8896 & 0.002 & 0.5791 & 5.7 \\
\hline 735 & 0.2824 & 0.002 & 0.4703 & 4.0 \\
\hline
\end{tabular}

